\title{
Ear, Nose, and Throat
}

\author{
Kara D. Meister and Anna H. Messner
}

\section{EARS}

\section{Preauricular Pits/Sinus (PPS)}

- Small indentations located anterior to the helix and superior to the tragus

- Can occur unilaterally ( $\sim 50 \%)$ or bilaterally $(\sim 50 \%)$

- Prevalence ranges between $1 \%$ and $10 \%$ depending on ethnicity

- Can occur in isolation with no increased risk of hearing impairment or renal issues

- Can be associated with hearing impairment and organ malformations

- Branchio-oto-renal (BOR) syndrome:

- Most common inherited syndrome causing hearing loss (autosomal dominant)

- Clinical presentation: preauricular pits, sensorineural hearing loss (SNHL), branchial cysts (may present as holes/pits in the side of the neck or as tags/pits in front of the ear), renal anomalies

- Beckwith-Wiedemann syndrome:

- Clinical presentation: macroglossia, asymmetric ear lobules or creases, omphalocele, Wilms tumor, hepatoblastoma.

K. D. Meister

Department of Otolaryngology-Head \& Neck Surgery, Pediatrics Division, Lucile Packard Children's Hospital, Stanford University, Stanford, CA, USA

A. H. Messner $(\square)$

Professor of Otolaryngology/Head \& Neck Surgery,

Baylor College of Medicine, Texas Children's Hospital,

Houston, TX, USA

e-mail: annamessner@stanford.edu
- Hearing loss can present later in childhood as conductive or mixed hearing loss

- PPS do not require surgical excision unless they are frequently draining or infected

- Passing of prenatal hearing screen should be confirmed in all patients

- Audiogram should be performed if there are other outer ear deformities or any evidence of genetic syndromes

- Wang et al. [1] suggest that a renal ultrasound be performed in children with ear anomalies accompanied by any of the following:

- Other known organ malformations

- Family history of deafness and auricular and/or renal malformation

- Maternal history of gestational diabetes mellitus

\section{Otalgia}

\section{Definition}

- Ear pain may be primary or referred in origin

\section{Clinical Presentation}

- Must consider potential sources of referred pain as well as sources of primary ear pain

- Primary: otitis externa, foreign body, acute otitis media (AOM), chondritis, ear canal laceration, mastoiditis

- Referred: temporomandibular joint (TMJ) disorders, larynx infection or pathology, deep neck space infection, cervical spine disorders

- Very common after oropharyngeal surgery (such as tonsillectomy) 


\section{Otitis Externa}

\section{Definition}

- Inflammation of the external auditory canal (EAC) due to bacterial (most commonly Pseudomonas aeruginosa, followed by Staphylococcus species) or fungal infections

\section{Clinical Presentation}

- Pain and tenderness with tragal pressure/pulling pinna; pruritic, erythematous, and edematous EAC; debris in the EAC; malodorous otorrhea

- Differential diagnosis: necrotizing otitis externa, otitis media with perforation, Ramsay Hunt syndrome, furuncle, atopic dermatitis, EAC foreign body, chondritis

\section{Treatment}

- Pain control and anti-inflammatories.

- Topical ear drops (ensured Pseudomonas coverage)

- Keep ears dry with water precautions (child either should swim with a neoprene headband to keep the ears dry or should not swim) and/ or with ear dryer (hair dryer)

- Ears drops of solution made of 50:50 white vinegar and rubbing alcohol can provide prophylaxis (if there's no tympanic membrane perforation). Commercial ear drops to prevent swimmer's ear are also available over the counter

\section{Indications for Ear, Nose, and Throat (ENT) Referral}

- Significant debris in the EAC-will require debridement.

- If unable to visualize the tympanic membrane due to canal edema, patient will require a temporary ear wick

- Immunocompromised or diabetic patient

\section{Foreign Body in the External Ear}

- Beads, insects, toys, popcorn, beans, and button batteries are common ear foreign bodies.
- Presentation: drainage, cough, pain, pruritus, child pulling at ear, incidentally noted during exam

- Most foreign bodies do not require emergent removal

- Emergent removal for button batteries.

- Indication for ENT referral:

- Presence of tympanic membrane perforation

- Foreign body wedged in the canal that cannot be grasped

- Trauma/bleeding in the ear canal

- Failed attempt at removal

\section{Hematoma of the External Ear (Pinna)}

- Commonly due to trauma

- Can cause avascular necrosis (blood supply lifted away from the cartilage by the hematoma) and permanent damage to the underlying cartilage (Fig. 18.1)

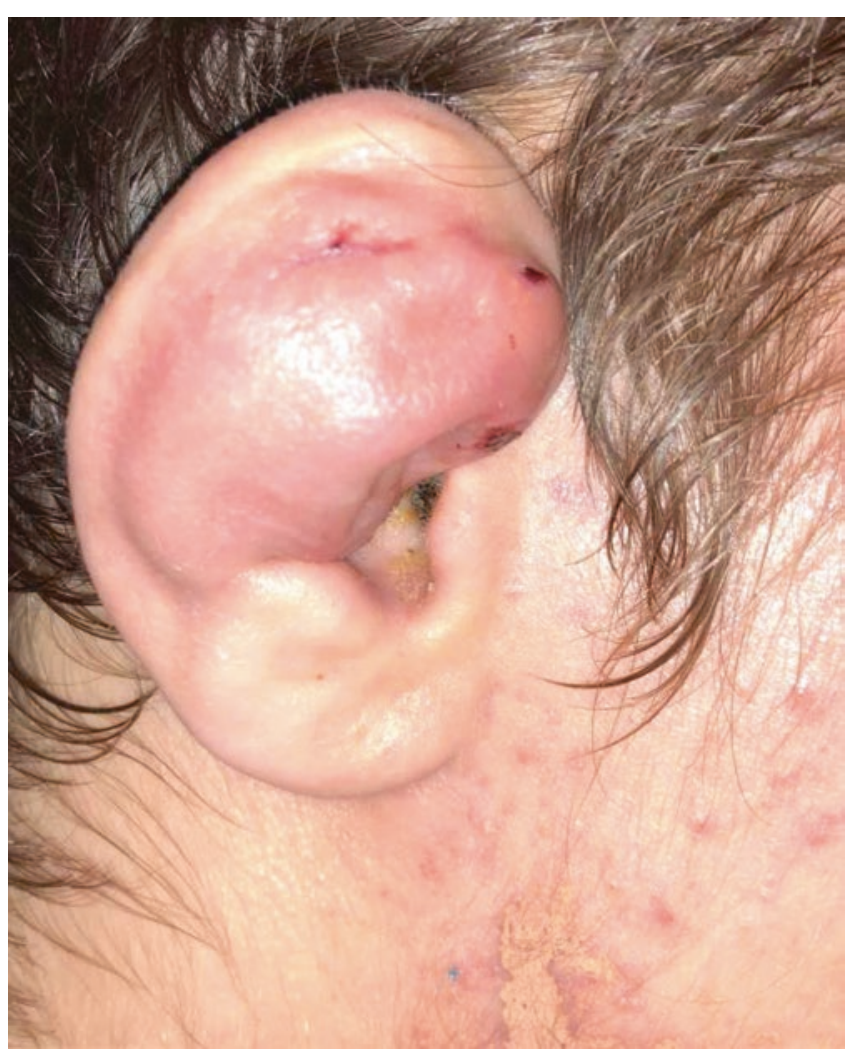

Fig. 18.1 Auricular hematoma of the right ear 


\section{Management}

- Urgent aspiration of hematoma to prevent pinna deformity (i.e., wrestler's ear or cauliflower ear)

- Pressure dressing applied after evacuation

- Close follow-up to monitor for reaccumulation

\section{Acute Otitis Media (AOM)}

\section{Background}

- Signs of an acute infection associated with middle effusion and inflammation (bulging tympanic membrane)

- $80 \%$ of children have at least one AOM before 1 year of age; $90 \%$ of children have at least two AOM by the age of 3

\section{Risk Factors}

- Age (6-18 months), positive family history of otitis media, daycare attendance, lack of breastfeeding, exposure to tobacco smoke, pacifier use/bottle propping, race/ethnicity (native Americans and the Inuit are at higher risk) [2]

\section{Common Pathogen}

- Bacterial: Streptococcus pneumoniae, nontypeable Haemophilus influenzae, Moraxella catarrhalis, and Streptococcus pyogenes (group A Streptococcus) are the most common causes.

- Viral: respiratory syncytial virus (RSV), picornavirus, coronavirus, influenza, adenovirus

\section{Clinical Presentation}

- Fever, irritability, apathy, anorexia, vomiting, diarrhea, otalgia, otorrhea, hearing loss

- Frequent nighttime awakening

\section{Diagnosis}

- Pneumatic otoscopy showing decreased tympanic membrane mobility remains the best method for diagnosing the presence of middle ear fluid
Management

- 2013 American Academy of Pediatrics guidelines [3]:

- Immediate antibiotic treatment for the following:

- Children $<6$ months of age

- Children with moderate to severe otalgia

- Otalgia lasting longer than $48 \mathrm{~h}$

- Temperature $>39^{\circ} \mathrm{C}\left(102.2^{\circ} \mathrm{F}\right)$

- Bilateral AOM and less than 24 months of age

- Antibiotic treatment or observation with pain control:

- 6 to 24 months of age with unilateral non-severe AOM.

$\circ>24$ months of age with unilateral or bilateral non-severe AOM.

- When observation is chosen, child must be followed up. Initiate antibiotics within 48-72 h should the child worsen or fail to improve

\section{Antimicrobial Therapy}

- First line: amoxicillin $(90 \mathrm{mg} / \mathrm{kg} /$ day divided twice a day) $\times 10$ days

- Second line, amoxicillin-clavulanate:

- Children who failed first-line therapy

- Children with increased risk of beta-lactam resistance

- Beta-lactam use within the past 30 days

- Concomitant purulent conjunctivitis (likely H. influenzae)

- Recurrent AOM unresponsive to amoxicillin

- For patients with hypersensitivity to penicillin:

- Macrolides

- Cefdinir, cefuroxime, ceftriaxone

\section{Complications}

- Intratemporal: conductive hearing loss (CHL), tympanic membrane perforation, ossicular erosion, labyrinthitis, facial nerve paralysis, mastoiditis, subperiosteal abscess, petrous apicitis, sigmoid sinus thrombosis 
- Intracranial: meningitis, epidural/subdural/ parenchymal abscess, cavernous sinus thrombosis, otitic hydrocephalus

\section{Recurrent Acute Otitis Media}

- 3 episodes in 6 months or 4 episodes in 1 year with 1 episode in the preceding 6 months [3]

- Associated conditions overlap with risk factors for Eustachian tube dysfunction: age $<2$ years, allergic rhinitis/sinusitis, mucociliary dysfunction, craniofacial abnormalities, immunodeficiency.

- Hallmark on physical exam is tympanosclerosis (a.k.a. myringosclerosis): white plaques on the tympanic membrane, which represent a form of scarring (Fig. 18.2)

- Recommendations to decrease risk: pneumococcal conjugate vaccine, annual influenza vaccine, exclusive breastfeeding for at least

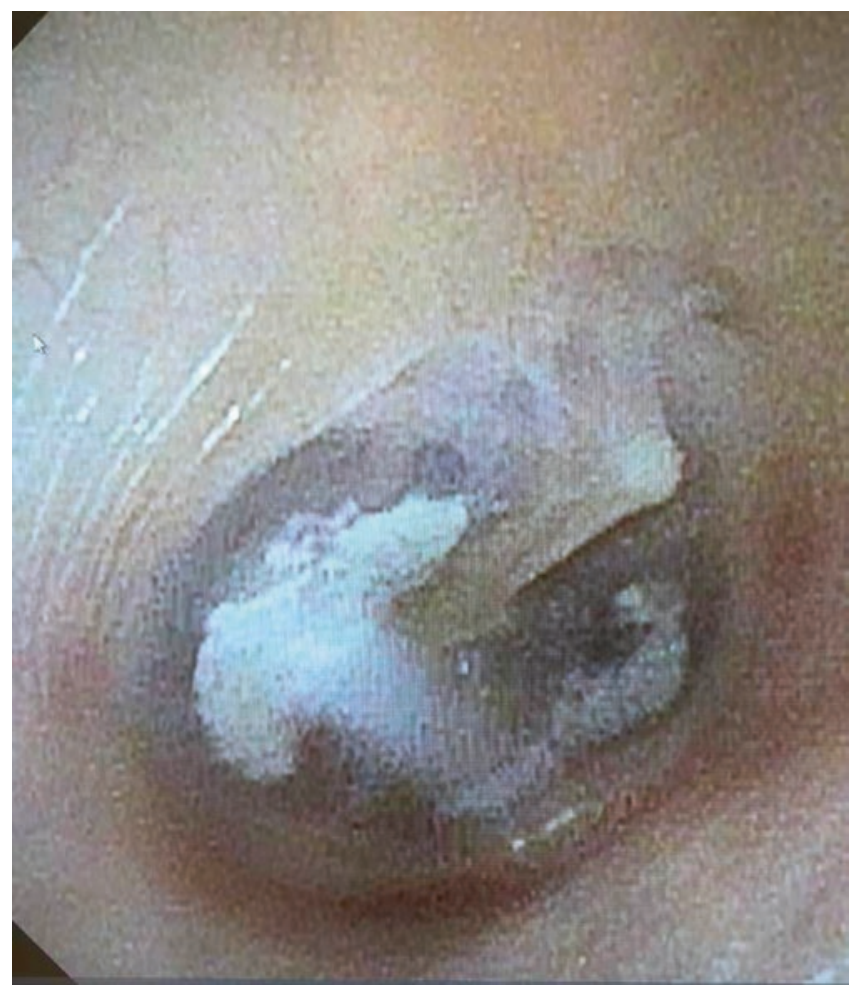

Fig. 18.2 Right tympanic membrane demonstrating tympanosclerosis
6 months, avoidance of tobacco smoke exposure

- Prophylactic antibiotics are not recommended

\section{Suggested Follow-Up}

- <2 years of age: 8-12 weeks after diagnosis/ treatment of AOM

- $<2$ years of age with language or developmental delay: 8-12 weeks after diagnosis/ treatment of AOM

- $>2$ years of age with no comorbidities/language or development delay: next routine visit

\section{Otitis Media with Effusion (OME)}

- Middle ear effusion without signs of acute infection

\section{Etiology}

- After AOM (typically):

- In the presence of Eustachian tube dysfunction in the absence of AOM

- It is estimated up to $90 \%$ of OME will resolve spontaneously within 3 months.

- $30 \%$ to $40 \%$ of patients will have recurrent episodes of OME.

- Most common cause of pediatric hearing loss.

- Frequency decreases as children age.

- More common in patients with Eustachian tube dysfunction:

- Craniofacial abnormalities (cleft palate and others): weak Eustachian tubes secondary to palate abnormalities

- Mucociliary dysfunction: primary ciliary dyskinesia

- Concurrent blockage of the Eustachian tube opening: adenoidal hypertrophy, allergic rhinitis/sinusitis

\section{Investigations}

- Hearing evaluation:

- Children with OME > 3 months 
- Children at risk of speech, language, and learning delay

- Speech language evaluation:

- In children at risk of speech, language, and learning delay

\section{Treatment}

- Observation, "watchful waiting"

- In children with OME with low risk of speech, language, and learning delays with speech awareness thresholds showing hearing loss less than $20 \mathrm{dBs}$.

- Monitor every 3 months to ensure resolution of effusion

- Myringotomy and tympanostomy tube insertion

- See Indication for Myringotomy and Tympanostomy Tubes for Acute Otitis Media (AOM) and Otitis Media with Effusion (OME)

\section{Chronic Suppurative Otitis Media (CSOM)}

\section{Definition}

- Otorrhea (> 6 weeks or recurrent) from a middle ear and/or mastoid infection in the presence of tympanic membrane perforation (or ventilation tube)

\section{Common Pathogen}

- Mixed infections:

- Gram-negative bacilli (Pseudomonas, Klebsiella, Proteus, Escherichia coli)

- Staphylococcus aureus

- Anaerobes

\section{Clinical Presentation}

- Otorrhea, TM perforation, inflamed middle ear mucosa, conductive hearing loss

\section{Treatment}

- Keep the ear clean and dry:

- Water precautions (avoid getting water in ear)
- Refer to otolaryngology if debridement is required.

- Topical antimicrobials/corticosteroids (must cover Pseudomonas and methicillin-resistant Staphylococcus aureus [MRSA]):

- If topical antibiotics failed, consider systemic antibiotics (broad spectrum covering Pseudomonas and MRSA)

\section{Indication for Myringotomy and Tympanostomy Tubes for Acute Otitis Media (AOM) and Otitis Media with Effusion (OME) [4, 5]}

- Bilateral myringotomy and tympanostomy tubes are indicated for a patient with the following:

- Bilateral OME for 3 months or more and documented hearing difficulties

- Unilateral or bilateral OME for 3 months and symptoms likely related to OME, for example, vestibular symptoms, poor school performance, behavioral difficulties, ear discomfort, and decreased quality of life

- Recurrent AOM and unilateral or bilateral middle ear effusion at the time of assessment

- At-risk children (such as those having craniofacial abnormalities or having only one hearing ear), with unilateral or bilateral OME that is unlikely to resolve quickly as reflected by a type B tympanogram (flat) or persistent effusion for 3 months or longer

- Complications of Tympanostomy Tubes

- Tube otorrhea (most common)

- Blockage of the tube

- Granulation tissue formation

- Displacement of the tube in the middle ear

- Tympanic membrane changes: myringosclerosis, atrophy, atelectasis, retraction pocket

- Persistent tympanic membrane perforation (may require surgical repair)

- Anesthesia-related complications 


\section{Acute Mastoiditis}

\section{Background}

- Suppurative infection of the middle ear (acute otitis media) that spreads to the mastoid cavity, resulting in osteitis of the mastoid bone

- May become purulent and lead to bony breakdown within the mastoid bone (acute coalescent mastoiditis)

\section{Common Presentation}

- Erythema, tenderness, and edema over the mastoid bone (postauricular region)

- Protuberant ear

- Fever, adenopathy, otitis media

\section{Assessment}

- Complete blood count (CBC), inflammatory markers, audiometric evaluation

- Imaging: computed tomography (CT) of the temporal bones (look for bony breakdown within the mastoid suggestive of coalescence)

- If there's any concern for intracranial complication, use of contrast is recommended.

\section{Treatment}

- Immediate otolaryngology consultation

- Systemic antibiotics (usually requires intravenous antibiotics)

- Possible myringotomy (tympanocentesis/culture) and ventilation tube (use topical antimicrobial if the tube is present)

- Cortical mastoidectomy for cortical erosion of the mastoid or intracranial complications

\section{Cholesteatoma}

\section{Definition}

- Squamous epithelium in the middle ear and mastoid cavities (misnomer as there's no cholesterol)

- Risk of leading to recurrent infections, as well as bone and soft tissue erosion
Types

- Congenital

- Presents as a white mass, most often in the anterior-superior middle ear space with an intact tympanic membrane

- Acquired

- Squamous epithelium which enters the middle ear via the retraction pocket (invagination), migration through tympanic membrane perforation, or iatrogenic implantation

\section{Clinical Presentation}

- Conductive hearing loss

- Persistent otorrhea

- Tympanic membrane retraction pocket filled with squamous epithelial debris/crusts

- Possible whitish mass behind the TM (not always seen)

\section{Complications}

- Erosion/destruction of the ossicular chain, chronic otitis media, labyrinthine fistula, intracranial complications/abscess, facial nerve paralysis

\section{Treatment}

- Otolaryngology consultation is mandatory.

- Requires surgery (tympanomastoidectomy, possible ossicular chain reconstruction)

- Long-term follow-up required by otolaryngology

\section{Labyrinthitis}

- Extremely rare in children

- Bacterial or viral invasion into the inner ear/ cochlear labyrinth; may be associated with permanent hearing loss, vestibular dysfunction, or meningitis

\section{Clinical Presentation}

- Vertigo, hearing loss, tinnitus, possible middle ear infection: 
- Labyrinthitis causes vertigo and hearing loss, whereas vestibular neuritis causes vertigo but spares hearing.

\section{Diagnosis}

- Clinical presentation, often preceded by an upper respiratory infection (URI)

- Obtain an urgent audiogram (sensorineural hearing loss)

\section{Treatment}

- Treat underlying infectious process:

- Bacterial (S. pneumoniae, H. influenzae, $M$. catarrhalis): systemic antibiotics

- Viral (cytomegalovirus [CMV], mumps, varicella-zoster virus, rubeola, influenza, parainfluenza, etc.): bed rest and hydration

- +/- Myringotomy/ventilation tube if acute otitis media is present

\section{Vertigo}

\section{Definition}

- Illusion of rotational, linear, or tilting movement (i.e., "spinning," "turning") of the patient or their surroundings

\section{Types of Vertigo}

- Central/systemic:

- Vascular (i.e., migraines, autoimmune disorders, stroke); anemia; brain tumor; medications, toxin, and chemotherapy; neurologic disorders (i.e., seizures, multiple sclerosis); metabolic disorders (i.e., thyroid disease, diabetes); anxiety and panic attack

- Peripheral (related to the ear):

- Benign positional vertigo of childhood (most common), vestibular neuritis due to viral infections (Epstein-Barr virus [EBV] most common), perilymph fistula (abnormal connection between the inner ear and middle ear), trauma to the vestibular system/concussion, Ménière disease, cerebellopontine angle tumors/acoustic neuroma

\section{Physical Exam}

- Vital signs

- Head and neck: complete exam, inspection of the middle ear/TM, pneumatic otoscopy

- Neurologic: complete cranial nerve exam, extraocular movements/nystagmus, coordination (finger-to-nose testing), gait, Romberg's test, gross vision testing

- Audiometric evaluation

\section{Treatment}

- Varies based on etiology.

- Refer to an otolaryngologist if suspicious of peripheral cause of vertigo; consider neurology referral if suspicious of central cause of vertigo

\section{Benign Paroxysmal Vertigo of Childhood}

\section{Definition}

- Most common peripheral vestibular disorder, typically self-limiting, can be recurrent

- Distinctly different from benign paroxysmal positional vertigo (BPPV), which is common in adults but not in children

\section{Causes}

- Spontaneous, posttraumatic, post-viral

- Thought to be a migraine equivalent

\section{Clinical Presentation}

- Brief recurrent episodes of vertigo lasting seconds to less than $1 \mathrm{~min}$

- Can be pale and sweaty, vomits during an episode and then appears back to normal

- No hearing loss or tinnitus

\section{Diagnosis}

- Clinical history essential; physical exam and vestibular testing usually normal:

- Rule out other more sinister causes of vertigo as directed by history and exam.

- Often a family history of migraines. 


\section{Treatment}

- Usually self-limiting

- Counsel on risk of typical migraine headaches later in life

\section{Meniere Disease}

\section{Background}

- Rare in children, but the prevalence ranges from $1.5 \%$ to $4 \%$ among children diagnosed with vertigo

\section{Clinical Presentation (Triad)}

1. Episodic vertigo (minutes to hours)

2. Episodic fluctuating sensorineural hearing loss (typically unilateral)

3. Tinnitus $+/-$ aural fullness in the affected ear

\section{Diagnosis}

- Clinical.

- Obtain an audiogram at time when patient reports hearing loss.

\section{Management}

- Refer to an otolaryngologist if suspicious of Meniere disease

\section{HEARING LOSS \\ AND AUDIOLOGY}

\section{Three Main Types of Hearing Loss}

- Conductive hearing loss (CHL):

- Normal bone conduction threshold with abnormal air conduction thresholds

- Presence of an air-bone gap (ABG)

- Indicative of a middle ear issue, for example, abnormalities with the tympanic membrane, ossicles, or middle ear space (i.e., effusion; Fig. 18.3)

- Sensorineural hearing loss (SNHL):

- When the air conduction is the same as the bone conduction with both showing abnormal hearing thresholds, this is suggestive of an inner ear issue resulting in sensori-

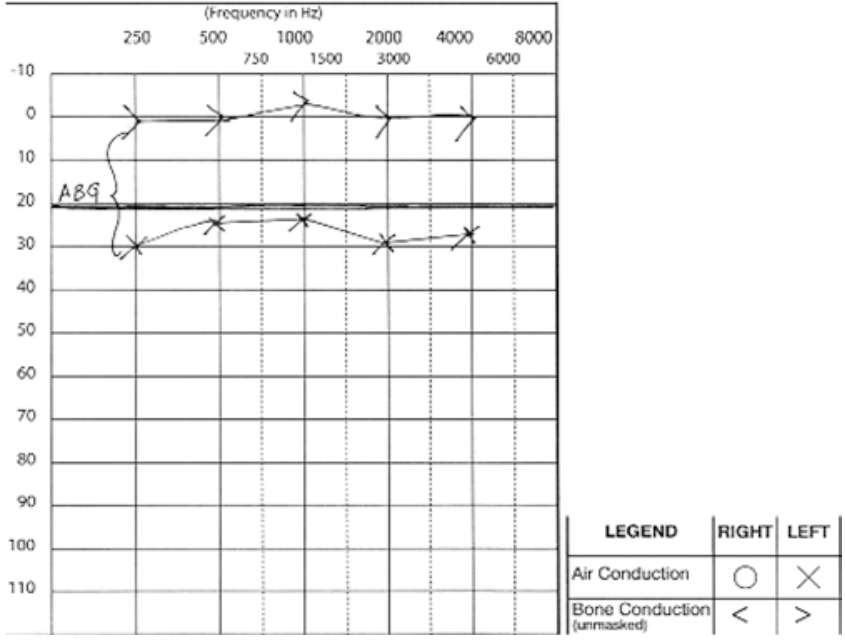

Fig. 18.3 Audiogram of mild conductive hearing loss

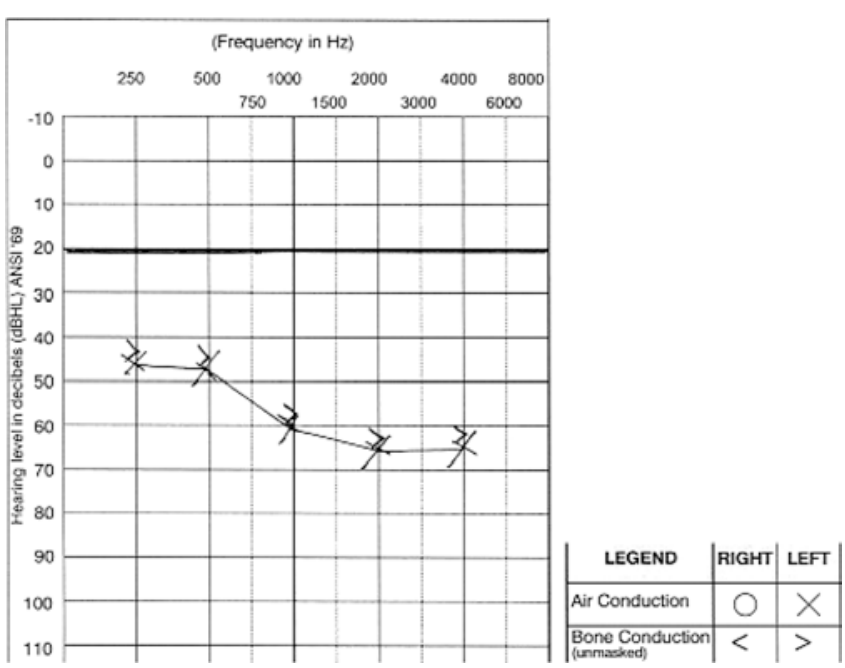

Fig. 18.4 Audiogram of moderate to moderate to severe sensorineural hearing loss

neural hearing loss (e.g., damage to cochlear, neural pathways and others)

- No ABG (Fig. 18.4)

- Mixed hearing loss (CHL+SNHL):

- Presence of conductive hearing loss and sensorineural hearing loss at the same time (Fig. 18.5)

\section{Congenital Hearing Loss}

- Congenital hearing loss can be divided into two main categories, environmental and genetic. This is further described in Table 18.1 [6] 


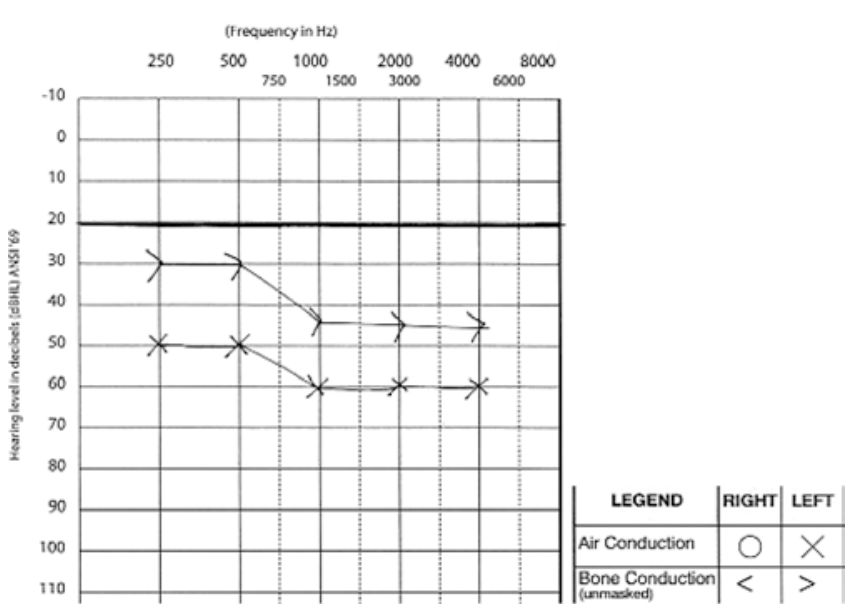

Fig. 18.5 Audiogram of mild to moderate sensorineural hearing loss with $\sim 20 \mathrm{dBs}$ conductive hearing loss

Table 18.1 Causes of congenital hearing loss [6]

\begin{tabular}{|c|c|c|}
\hline \multirow{7}{*}{$\begin{array}{l}50 \% \\
\text { environmental }\end{array}$} & \multicolumn{2}{|c|}{ Cytomegalovirus (CMV) } \\
\hline & \multicolumn{2}{|l|}{ Neonatal icterus } \\
\hline & \multicolumn{2}{|l|}{ Meningitis } \\
\hline & \multicolumn{2}{|l|}{ Rubella } \\
\hline & \multicolumn{2}{|l|}{ Prematurity } \\
\hline & \multicolumn{2}{|l|}{ Ototoxicity } \\
\hline & \multicolumn{2}{|l|}{ Other infections } \\
\hline \multirow[t]{14}{*}{$50 \%$ genetic } & \multirow{13}{*}{$\begin{array}{l}30 \% \\
\text { syndromic }\end{array}$} & Autosomal recessive: \\
\hline & & Usher syndrome \\
\hline & & Pendred syndrome \\
\hline & & Jervell and Lange- \\
\hline & & Nielsen syndrome \\
\hline & & Alport syndrome \\
\hline & & (sometimes X-linked) \\
\hline & & Autosomal dominant: \\
\hline & & Waardenburg syndrome \\
\hline & & Stickler syndrome \\
\hline & & $\begin{array}{l}\text { Branchio-oto-renal } \\
\text { syndrome }\end{array}$ \\
\hline & & Treacher Collins \\
\hline & & syndrome \\
\hline & $\begin{array}{l}70 \% \\
\text { nonsyndromic }\end{array}$ & Connexin mutations \\
\hline
\end{tabular}

\section{Genetic Syndromic Hearing Loss}

- More than 500 syndromes are associated with hearing loss. The most common are listed as follows:

\section{Autosomal Dominant}

- Waardenburg syndrome: SNHL, hypertelorism, pigmentary abnormalities
- Stickler syndrome: SNHL, ocular abnormalities (myopia, retinal detachment), Marfanoid habitus, Pierre Robin sequence

- Branchio-oto-renal syndrome: mixed hearing loss (sensorineural and conductive hearing loss), pinna deformities, preauricular or neck pits/fistulas/tags, kidney abnormalities

- Treacher Collins syndrome (mandibulofacial dysostosis): CHL (malformed ossicles), aural atresia/stenosis, zygomatic/mandibular hypoplasia

- Others: neurofibromatosis type II, Apert syndrome (acrocephalosyndactyly), Crouzon syndrome (craniofacial dysostosis)

\section{Autosomal Recessive (mnemonic 'PUJ")}

- Usher syndrome:

- Leading cause of deafness and blindness

- SNHL, blindness (retinitis pigmentosa), vestibular dysfunction

- Pendred syndrome: SNHL, goiter, enlarged vestibular aqueducts

- Jervell and Lange-Nielsen syndrome: SNHL, cardiac defects (prolonged QT), syncope, sudden death

X-Linked

- Alport syndrome: X-linked, hearing loss, progressive nephritis, occasional ocular lesions

\section{Genetic Nonsyndromic Hearing Loss}

\section{Connexin Mutations}

- Most common cause of hereditary nonsyndromic hearing loss.

- Connexin 26 mutations (GJB2 gene) account for $\sim 80 \%$

- Testing done as part of the workup for bilateral SNHL.

\section{Universal Newborn Hearing Screening}

- Implemented across all states in the USA and provinces in Canada.

- Tests hearing with otoacoustic emission (OAE) screening or with an automated auditory 
brainstem response $(A B R)$ shortly after birth (usually before the neonate leaves the hospital)

- Any infant who fails the initial screen should be referred to an audiologist for a full evaluation no later than 3 months of age.

- For all children in whom hearing loss is established by full audiologic evaluation, intervention must begin as soon as possible and no later than 6 months of age

\section{Clues to Hearing Loss in a Child Visit}

- Speech delay

- Social and behavioral challenges

- A child asking people to repeat themselves, not hearing instructions

- Listening to loud television or music

- Clumsiness-may be a clue to middle ear fluid

\section{Pediatric Audiometric Testing}

\section{Evoked Otoacoustic Emission (OAE)}

- OAE detects the sound coming from the cochlea in response to clicks or tones.

- OAE affected by external or middle ear debris (high false-positive rate)

- Used for all ages

- No infant cooperation is required.

\section{Auditory Brainstem Response (ABR)}

- ABR measures the electroencephalographic waveform response from the vestibulocochlear nerve to higher central nervous system auditory centers.

- ABR minimally affected by external or middle ear debris.

- Can be used at any age.

- Patient must be asleep or very still-may require sedation.

- Often used to confirm abnormal OAE results.

\section{Testing Methods}

Behavioral Observation Audiometry (BOA)

- Birth-6 months of age
- Sound presented via speakers. Skilled examiner observes for patient response (i.e., startling or head turning toward sound)

- Grossly assessed auditory thresholds of "better" ear (tests both ears at the same time)

\section{Visual Response Audiometry (VRA)}

- Six months to 3 years of age.

- Toddler encouraged to look for auditory stimulus (i.e., lights, toys, motion for reinforcement)

- Each ear may be tested individually-potential to provide complete audiogram

\section{Play Audiometry}

- 3 to 5 years of age.

- Child performs tasks in response to auditory stimulus (e.g., "Pick up a block and place it in the bucket when you hear the beep")

- Each ear is tested individually, frequency specific

\section{Conventional Audiometry}

- 4 to 6 years of age and older

- Child instructed to push a button or raise a hand when a tone is heard

- Complete audiogram, ear specific, frequency specific

\section{Hearing Loss Classification}

- Classified by hearing threshold levels (may vary slightly based on sources):

- Normal: < $19 \mathrm{~dB}$

- Mild: 20-40 dB

- Moderate: 41-55 dB

- Moderate to severe: $56-70 \mathrm{~dB}$

- Severe: 71-90 dB

- Profound: $91 \mathrm{~dB}$

\section{Tympanometry}

- Age: all ages except newborn.

- Detects the mobility of TM and external auditory canal volumes.

- Normal canal volumes range between 0.2 and $1.5 \mathrm{ml}$ :

- Type A

- Normal peak between -150 and +50 deka pascals 
- Type B

○ Flat, no peak

- Suggestive of:

- Middle ear effusion (normal to low volumes)

- Tympanic membrane perforation (high canal volumes)

- Patent ventilation tube (high canal volumes)

- Type C

- Peak negatively shifted $(<-150)$

- Suggestive of a retracted tympanic membrane or Eustachian tube dysfunction

\section{Patterns of Hearing Loss}

\section{Interpreting an Audiogram}

- $Y$-axis $=$ hearing level in decibels $(\mathrm{dBs})$ or the "loudness" of sound

- $X$-axis = frequency of sound presented measured in Hertz (low pitch to high pitch)

- " $\mathrm{x} "=$ responses from the left air conduction line

- ">" = responses from the left bone conduction line

- $\mathrm{ABG}=$ difference between air conduction and bone conduction lines

\section{Common Clinical Scenarios}

- Tympanic membrane perforation (Fig. 18.6):

- Audiometric findings:

- ABG

- Flat tympanogram

- High canal volumes

- Mild conductive hearing loss

- Middle ear effusion (Fig. 18.7):

- Audiometric findings:

- $\mathrm{ABG}$

- Flat tympanogram

- Low or normal canal volumes

- Mild conductive hearing loss

- Ototoxicity (Fig. 18.8):

- Ototoxic medications cause hearing loss by damaging the hair cells within the cochlea, resulting in sensorineural hearing loss, primarily in the high frequencies.

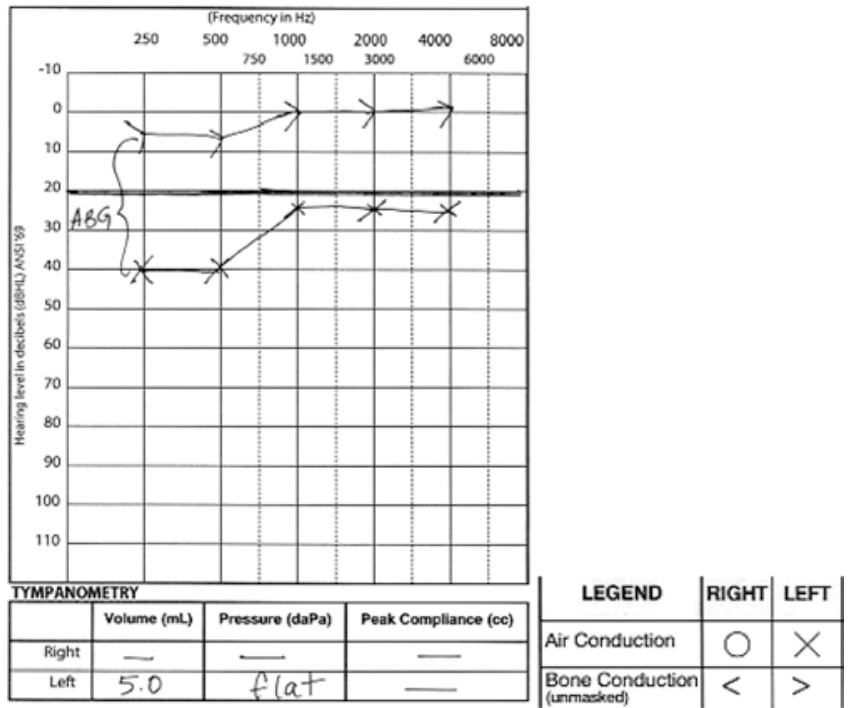

Fig. 18.6 Audiogram of tympanic membrane perforation

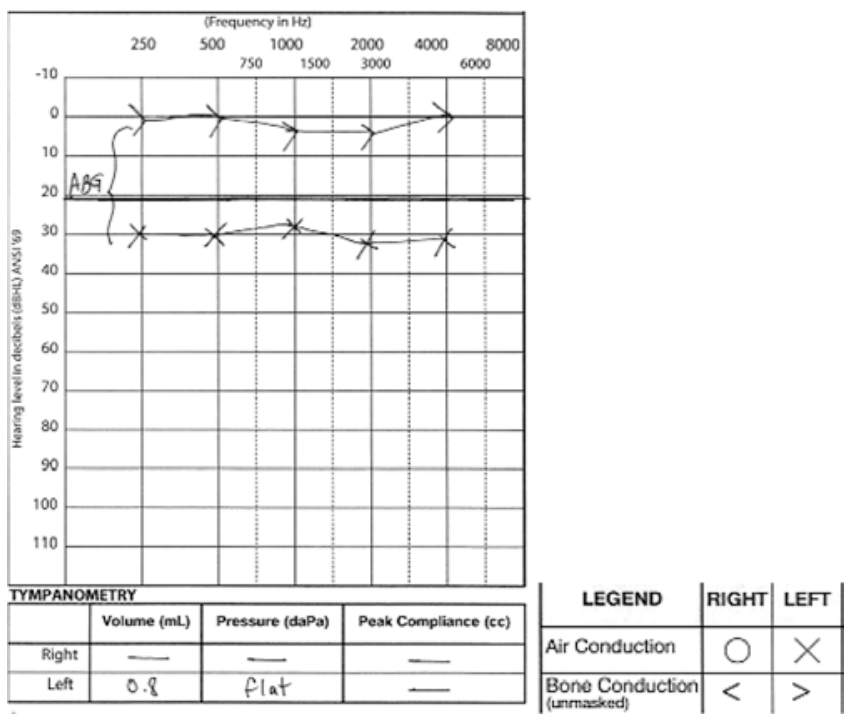

Fig. 18.7 Audiogram of middle ear effusion

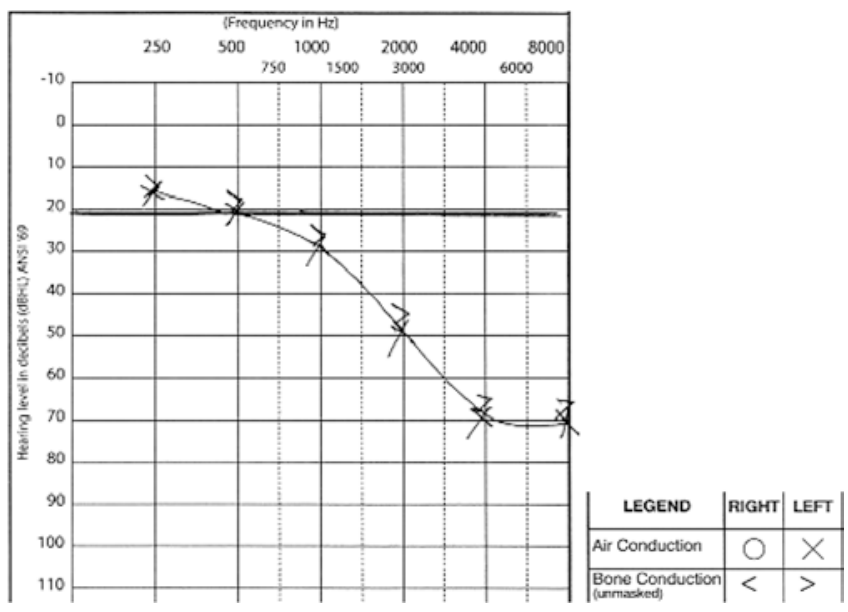

Fig. 18.8 Audiogram of ototoxicity 


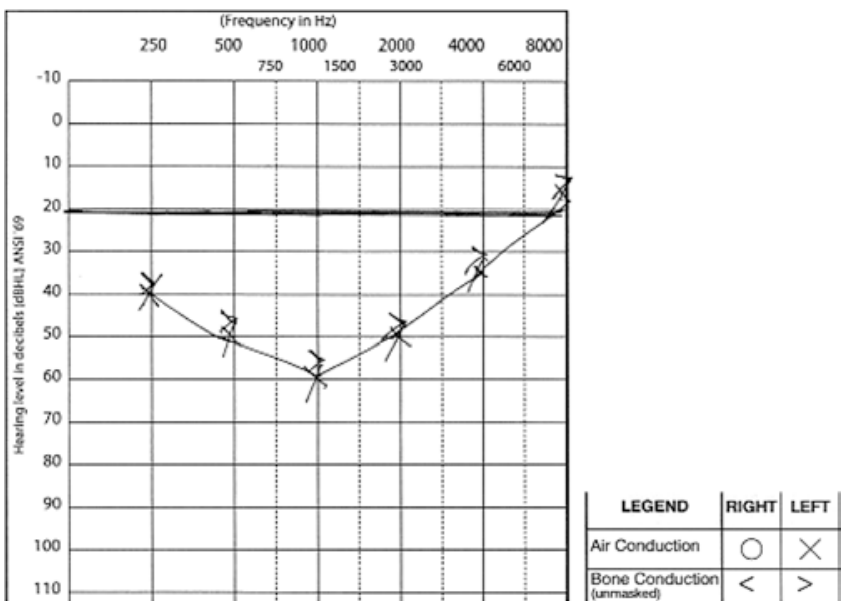

Fig. 18.9 Audiogram of hereditary hearing loss

- Most commonly caused by cisplatin/ carboplatin.

- Audiometric findings:

- High-frequency sensorineural hearing loss (moderate to moderate to severe)

- No ABG

- Normal tympanogram and volumes (typically)

- Hereditary hearing loss (Fig. 18.9):

- Cookie bite (U-shape) pattern of sensorineural hearing loss

- No ABG, normal tympanogram, normal canal volumes

\section{Sound Amplification Devices}

- Early identification and intervention is required to maximize hearing and speech development, as well as to achieve developmental milestones.

- Refer to an otolaryngologist when an abnormal hearing screen is identified.

- Hearing interventions are dependent on type and severity of hearing loss.

\section{Hearing Aids}

- Non-implantable external hearing device that amplifies frequency-specific sounds.

- Used for unilateral or bilateral CHL, SNHL, and mixed hearing losses.
- Wide variety available, depending on hearing needs and preferences.

- Fitting and programming processes are complex and completed by an audiologist

\section{Bone-Anchored Hearing Aid (Osseo-integrated Auditory Implant)}

- Titanium implant surgically placed in mastoid bone behind the ear.

- Sound processor is placed externally on the implant and conducts sounds via bone contact and vibration.

- Primarily used in patients with unilateral or bilateral CHL with congenital ear malformation (i.e., atresia, canal stenosis)

\section{Cochlear Implants}

- Convert sound to an electrical signal that stimulates the cochlear nerve.

- External component captures sound and converts it to an electrical signal.

- Internal component delivers frequencyspecific electrical signal to the cochlear nerve

- Multiple cochlear implant devices are available, depending on hearing loss pattern and patient preferences

- Cochlear implant criteria are very specific and include a multidisciplinary team (otolaryngologist, speech pathologist, audiologist, social worker, psychologist, etc.)

- In general, indicated for children (US Food and Drug Administration [FDA] approved for 12 months of age or older, though children are often implanted younger than 12 months of age) with pre- or postlingual severe to profound bilateral high-frequency SNHL

- Fitting and programming is a complex process performed by a specialized cochlear implant audiologist and requires multiple audiology visits

- There is increasing evidence regarding the benefits of binaural hearing 


\section{NOSE AND NASOPHARYNX}

\section{Choanal Atresia}

\section{Background}

- Congenital obstruction of the choana (posterior nasal aperture — connects the nasal cavity to the nasopharynx):

- Diagnosis is suspected with the inability to pass a $5 \mathrm{~F}$ suction catheter through the nose into the nasopharynx

- It may be mixed (60\%), bony (30\%), or membranous (10\%) (CT scan can help to confirm and identify the type of atresia)

- Unilateral or bilateral 2:1 ratio.

- Syndromic association with congenital anomalies is associated with unilateral choanal atresia $50 \%$ of the time, and there is a syndromic association $75 \%$ of the time if the atresia is bilateral:

- Can be associated with syndromes (e.g., coloboma of the eye, heart defects, atresia of the nasal choanae, retardation of growth and/or development, genital and/or urinary abnormalities, and ear abnormalities (CHARGE) and deafness, trisomy 21, trisomy 18, Treacher Collins syndrome, Apert syndrome, Crouzon syndrome, velocardiofacial syndrome)

\section{Clinical Presentation}

- Bilateral:

- Severe respiratory distress at birth, cyclical cyanosis-pink with crying, cyanotic when not crying

- Requires immediate oral airway or intubation; refer to otolaryngology once airway is secured for surgical repair in the first few days of life

- Patients with bilateral atresia and those with syndromes (e.g., CHARGE) are at greater risk of restenosis
- Unilateral:

- Identified at birth due to inability to pass $6 \mathrm{~F}$ catheter or later in childhood with unilateral symptoms of rhinorrhea, decreased nasal patency, or stertor

- Surgical repair based on symptoms and growth, often around 1 year of age and nearly all before 4 years of age

\section{Epistaxis}

\section{Background}

- In children, $90 \%$ of epistaxis occurs from the anterior septum (Kiesselbach plexus)

- Posterior epistaxis is rare in children and should prompt further evaluation.

- Most common causes: trauma (i.e., nose picking), mucosal irritation and drying, foreign body, and medications (e.g., nasal steroids)

\section{Other Causes}

- Tumors, e.g., juvenile nasopharyngeal angiofibroma (occurs in pubescent males), pyogenic granuloma

- Vascular malformation: hemangioma, telangiectasia, Osler-Weber-Rendu syndrome (+ family history)

- Bleeding diathesis: von Willebrand disease, leukemia, liver disease

\section{Management}

- Usually self-limiting with application of constant pressure for $5 \mathrm{~min}$ by squeezing the sides of the nose shut

- Discourage nose picking/rubbing.

- Avoid mucosal dryness-humidifier in the bedroom; apply small amount of nasal lubricant to the anterior septum

- If severe, it will need IV access and formal nasal packing +/- airway management +/hemodynamic resuscitation

- Refer to an otolaryngologist if suspicious for a foreign body, tumor, recurrent epistaxis, or severe epistaxis 


\section{Nasal Trauma}

\section{Background}

- Nasal fractures are the most common facial fracture in children (followed by the mandible)

- Most commonly secondary to falls, sporting collisions, motor vehicle accidents.

\section{Presentation}

- External nasal deformity, nasal obstruction, epistaxis, anosmia, septal deviation, edema, bruising

\section{Assessment}

- Pediatric Advanced Life Support (PALS); rule out injuries to the cervical spine, central nervous system, and chest, orbit/vision problems, midface stability, malocclusion, presence of telecanthus, cerebrospinal fluid leak, etc.

- Nasal x-rays not useful.

- Must evaluate for septal hematoma/abscess.

\section{Clinical Presentation}

- Boggy asymmetrical swelling of the nasal septum not responsive to topical vasoconstriction

- Management of nasal hematoma: requires urgent drainage by an otolaryngologist +/bolster dressing to prevent nasal cartilage necrosis

\section{Management}

- If there's cosmetic deformity +/- functional issues (e.g., decreased nasal patency), refer to otolaryngology for reduction of nasal fracture.

\section{SINUSES}

\section{Acute Rhinosinusitis}

\section{Definitions}

- Sinusitis: mucosal inflammation of paranasal sinuses typically caused by a viral illness
Table 18.2 Clinical characteristics of viral vs. bacterial rhinosinusitis

\begin{tabular}{|c|c|c|}
\hline $\begin{array}{l}\text { Clinical } \\
\text { feature }\end{array}$ & Viral rhinosinusitis & $\begin{array}{l}\text { Bacterial } \\
\text { rhinosinusitis }\end{array}$ \\
\hline Fever & $\begin{array}{l}\text { Absent or occurs } \\
\text { early (first } 24 \mathrm{~h} \text { )- } \\
\text { low grade, resolves } \\
\text { in } 2 \text { days }\end{array}$ & $\begin{array}{l}\text { Present, }>39^{\circ} \mathrm{C} \\
\left(102^{\circ} \mathrm{F}\right) \times 3 \text { days, may } \\
\text { develop or recur on } \\
\text { days } 6-7 \text { of illness }\end{array}$ \\
\hline $\begin{array}{l}\text { Nasal } \\
\text { discharge }\end{array}$ & $\begin{array}{l}\text { Peaks on days 3-6 } \\
\text { and then improves }\end{array}$ & $\begin{array}{l}\text { Fails to improve or } \\
\text { worsens }\end{array}$ \\
\hline Cough & $\begin{array}{l}\text { Peaks on days 3-6 } \\
\text { and then improves }\end{array}$ & $\begin{array}{l}\text { Fails to improve or } \\
\text { worsens }\end{array}$ \\
\hline $\begin{array}{l}\text { Ill- } \\
\text { appearance }\end{array}$ & Absent & $\begin{array}{l}\text { If severe or } \\
\text { complicated }\end{array}$ \\
\hline $\begin{array}{l}\text { Severe } \\
\text { headache }\end{array}$ & Absent & $\begin{array}{l}\text { If severe or } \\
\text { complicated }\end{array}$ \\
\hline $\begin{array}{l}\text { Clinical } \\
\text { course }\end{array}$ & $\begin{array}{l}\text { Peaks on days 3-6 } \\
\text { and then improves }\end{array}$ & $\begin{array}{l}\text { > } 10 \text { days, without } \\
\text { improvement }\end{array}$ \\
\hline
\end{tabular}

- Acute bacterial rhinosinusitis (ABRS): sinusitis secondary to bacterial infection

- Acute: < 90 days

\section{Risk Factors}

- URI

- Daycare

- Allergic rhinitis

- Anatomic anomalies (e.g., septal deviation)

\section{Presentation}

- Congestion, purulent rhinorrhea, tenderness over sinuses:

- Clinical presentation may be variable based on the pathogen, as described in Table 18.2.

- Virology/microbiology:

- Viruses: rhinovirus, parainfluenza, influenza, adenovirus

- Bacteria: S. pneumoniae, H. influenzae, $M$. catarrhalis

- Risk of antimicrobial resistance [7]:

- Age $<2$ years, daycare antibiotics in the past month, hospitalization within 5 days

\section{Treatment}

- Over-the-counter cold medications or decongestants (either systemic or intranasal) are not recommended for children under 12 years of age. 
- Supportive therapies: hydration, saline nasal rinses, acetaminophen/ibuprofen.

\section{Treatment of ABRS [8]}

- Saline nasal rinses.

- Antibiotics:

- First line: amoxicillin/clavulanic acid $45 \mathrm{mg} / \mathrm{kg}$ divided BID $\times 10-14$ days

- If at risk of resistance (see above), $90 \mathrm{mg} /$ $\mathrm{kg}$ divided BID $\times 10-14$ days

- Third-generation cephalosporins if there's penicillin hypersensitivity

- Surgery:

- No role in ABRS, unless there's evidence of complication (i.e., orbital or intracranial)

- Monitor for complications:

- Orbital

- Preseptal cellulitis, orbital cellulitis, subperiosteal abscess, orbital abscess, cavernous sinus thrombosis

- Intracranial

- Meningitis, epidural abscess, subdural abscess, parenchymal abscess, etc.

- Osteomyelitis (typically of frontal bones)

\section{Imaging}

- CT scan of the sinuses is only indicated for the following:

- If suspicious for sinusitis complications (e.g., orbital or intracranial)

- Failure of antibiotic treatment $\times 48 \mathrm{~h}$

- Immunocompromised patient

- Findings: opacification of sinuses, mucosal thickening, air-fluid levels

- Note: These findings are also present with the common cold.

\section{Chronic Sinusitis}

\section{Definition}

- Persistence of symptoms > 12 weeks.

- Symptoms include nasal congestion, facial pressure, nasal obstruction, rhinorrhea/postnasal drip, and altered sense of smell.

\section{Risk Factors}

- Young age (developing immune system), URI, ciliary dysfunction, allergic rhinitis, gastroesophageal reflux disease (GERD), immune deficiency, cystic fibrosis

\section{Microbiology}

- Aerobes: S. pneumoniae, M. catarrhalis, H. influenzae, Staphylococcus aureus, Pseudomonas

- Anaerobes: Peptococcus, Peptostreptococcus, Bacteroides

\section{Diagnosis}

- Clinical diagnosis (imaging not required for diagnosis)

- Plain X-ray films are generally NOT helpful.

- CT scan indicated when failed medical management and surgical intervention is being considered.

\section{Treatment}

- Medical management:

- Saline nasal rinses.

- Antibiotics: amoxicillin-clavulanic acid $\times 3-4$ weeks.

- Topical nasal steroids.

- Consider treatment of gastroesophageal reflux disease (GERD) if suspicious.

- Surgical:

- Only considered if there's failure of longterm medical management.

- Adenoidectomy is first line of surgery.

- If symptoms are persistent following adenoidectomy and the patient continues to fail medical management, functional endoscopic sinus surgery (maxillary antrostomy and ethmoidectomy usually sufficient unless clinical symptoms or imaging suggests other sinuses involved) may be considered.

- Ancillary tests:

- If medical management fails, consider allergy testing if suspicious for allergies.

- If negative, consider workup for primary immunodeficiency disorder if suspicious. 


\section{Frontal Sinus Trauma}

- Rare in children, as frontal sinuses begin forming around 6-7 years of age.

- Associated with high-impact injury-must rule out cervical spine injuries and intracranial injury.

- May present with forehead lacerations or swelling, palpable frontal defect, pain, epistaxis, cerebrospinal fluid leak.

- CT scan optimal for identifying fractures, magnetic resonance imaging (MRI) considered in addition to assessing intracranial involvement.

- If frontal sinus fracture is present, consult otolaryngology for further management.

- Conservative or surgical depending on fracture pattern.

- If suspicious for intracranial involvement, consult neurosurgery.

\section{THROAT AND OROPHARYNX}

\section{Tonsillitis/Pharyngitis}

\section{Etiology}

- Infectious (most common), allergy, GERD [9]

- Viral (most common):

- Rhinovirus, coronavirus, adenovirus, herpes simplex virus (HSV), EBV, coxsackie virus

- Usually associated with symptoms of cough, sneezing, rhinorrhea, low-grade fever

- Bacterial (streptococci, pneumococci, $H$. influenzae):

- Bacteria are responsible for 5-10\% of pharyngitis.

- Group A b-hemolytic Streptococcus (GABHS) — most common bacterial cause [10]

- Usually associated with symptoms of highgrade fever, tonsillar/palatal petechiae, exudative tonsils, tender lymphadenopathy; rarely seen with cough or rhinorrhea; most common in children 5-12 years of age.

- GABHS pharyngitis should be treated to reduce the risk of rheumatic fever and scarlet fever.

- Other bacterial causes: syphilis, pertussis, gonorrhea, diphtheria, Fusobacterium.

- Untreated Fusobacterium infections may lead to Lemierre syndrome: thrombus of the internal jugular vein with potential for septic emboli.

\section{Symptoms}

- Sore throat, pain with swallowing, ear pain (referred), malaise, fever, oropharyngeal erythema, cervical lymphadenopathy

\section{Diagnosis}

- Based on history and physical exam

- Throat cultures, the diagnostic standard:

- A positive posttreatment culture represents the asymptomatic chronic carrier state (not a significant source for the spread of GABHS, no treatment needed)

- If there are recurrent symptoms and persistently positive culture, consider compliance failure, exposure, immunosuppression, and penicillin-resistant organism (consider second-line therapy, such as amoxicillin-clavulanate)

- GABHS rapid antigen test:

- Sensitivity/specificity: enzyme immunoassay in children, pooled sensitivity estimated at $86 \%$ and the pooled specificity estimated to be $92 \%$; for immunochromatographic assay in children, pooled sensitivity estimated at $88 \%$ and the pooled specificity estimated to be $86 \%$

- Throat culture should follow negative rapid antigen test when diagnosis of GABHS infection is strongly suspected.

- Monospot test (EBV)

- Streptococcal antibody titers not recommended 


\section{Treatment}

- Ensure airway safety.

- Supportive:

- Hydration, humidity, analgesia

- Antibiotics if bacterial infection is suspected (confirm with cultures):

- Treatment proposed to decrease symptoms within $\sim 16 \mathrm{~h}$, decreased rates of peritonsillar abscess (PTA) and retropharyngeal abscess.

- Modified Centor score may be used to help physicians decide which patients need no testing, throat culture/rapid antigen detection testing, or empiric antibiotics.

\section{Peritonsillar Abscess (PTA)}

\section{Definition}

- Peritonsillar space defined:

- Space between the palatine tonsil, superior constrictors, and tonsillar pillars

\section{Etiology}

- More common in adolescents

- Spread of infection from the tonsil

- Pathogens: aerobes (Streptococcus pyogenes, Staphylococcus aureus, Haemophilus influenzae, and Neisseria species) and anaerobes

\section{Clinical Presentation}

- Sore throat, painful swallowing, uvular deviation to the contralateral side (medialization), trismus, asymmetrical swelling on the soft palate, "hot potato" voice, fevers, referred otalgia.

- Symptoms are typically present for at least 3 days before abscess is formed; double worsening phenomenon is common.

\section{Diagnosis}

- History and physical examination.

- CT for atypical cases or if there are concerns for retropharyngeal/parapharyngeal space involvement.
Table 18.3 Clinical features of peritonsillar abscess vs. retropharyngeal space abscess

\begin{tabular}{|l|l|}
\hline Retropharyngeal abscess & Peritonsillar abscess \\
\hline$<6$ years old & Adolescent \\
\hline Fever, throat pain, neck stiffness & $\begin{array}{l}\text { Fever, throat pain, } \\
\text { trismus }\end{array}$ \\
\hline $\begin{array}{l}\text { Purulence of retropharyngeal } \\
\text { lymph node }\end{array}$ & $\begin{array}{l}\text { Purulence of tonsillar } \\
\text { fossa }\end{array}$ \\
\hline May need imaging studies & $\begin{array}{l}\text { Usually diagnosed } \\
\text { clinically }\end{array}$ \\
\hline
\end{tabular}

- Clinical features of PTA and retropharyngeal space abscess are outlined in Table 18.3.

- PTA and retropharyngeal abscess can occur concurrently.

\section{Management}

- Surgical incision and drainage.

- Antibiotic therapy (penicillin or clindamycin)

- Two or more PTAs may require a future tonsillectomy (bilateral) once infection resolves.

- "Quinsy tonsillectomy"-tonsillectomy at the time of infection may be considered in younger children but is rare in practice secondary to acute inflammation of the tonsils.

\section{Retropharyngeal Abscess}

\section{Definition}

- Space between pharyngeal constrictors and the alar fascia (skull base to the mediastinum)

\section{Etiology}

- Presents most commonly in children

- Spread of infection from the tonsils, sinuses, and/or nasopharynx

- Polymicrobial flora (most commonly Staphylococcus aureus, Streptococcus species, and anaerobes)

\section{Clinical Presentation}

- Fevers, "hot potato voice," painful swallowing, drooling, decreased neck range of motion (typically limited neck extension), trismus 


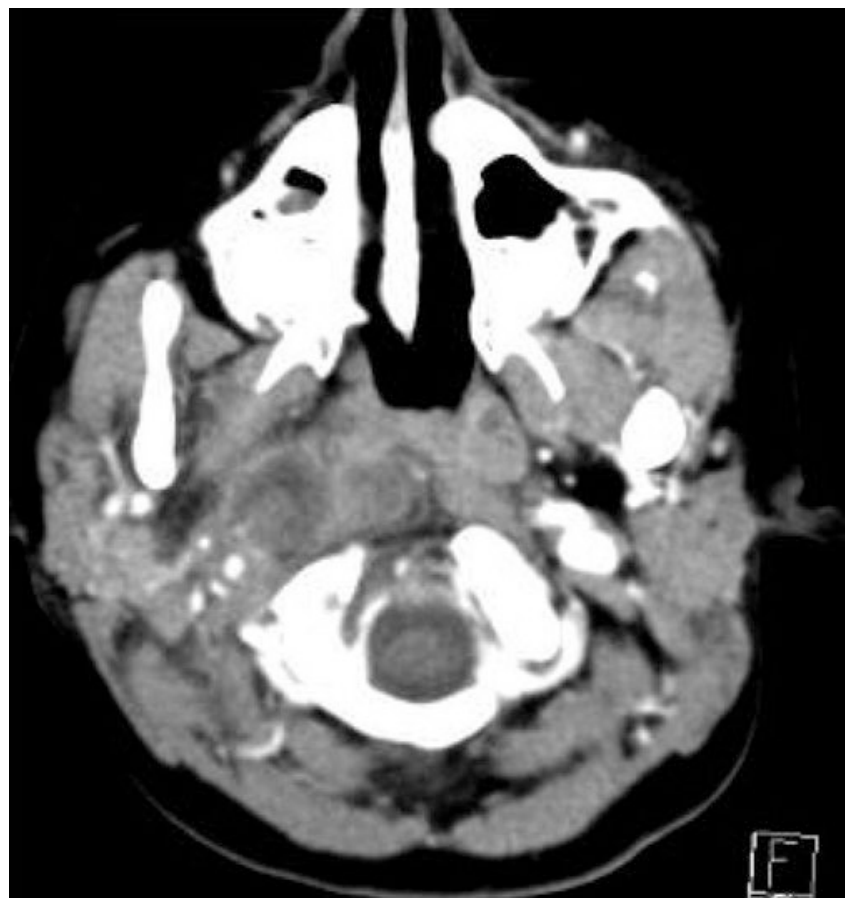

Fig. 18.10 Concurrent peritonsillar abscess on the right and retropharyngeal abscess in a 10-year-old boy as seen on axial computed tomography scan

possible if there's pterygoid inflammation, possible airway compromise/stridor if severe

\section{Diagnosis}

- Lateral neck radiograph: abnormally increased thickness of the prevertebral soft tissue (greater than half thickness of the adjacent vertebral body)

- CT scan with contrast useful for localization, extension, phlegmon, or abscess (Fig. 18.10)

\section{Treatment}

- Airway management if required and/or ongoing airway monitoring

- Hydration and analgesia

- Antibiotics (may consider third-generation cephalosporin, clindamycin, or ampicillin/ sulbactam for first line)

- Surgical drainage indicated for failed medical management, well-defined rim-enhancing abscess, systemic illness, and/or airway compromise

\section{Indication for Tonsillectomy \\ (+/- Adenoidectomy) $[11,12]$}

\section{Absolute Indications}

- Moderate to severe obstructive sleep apnea

- Suspicions of tonsillar malignancy, including posttransplant lymphoid proliferative disorder (PTLD)

\section{Relative Indications}

- Mild obstructive sleep apnea

- Recurrent tonsillitis-must meet criteria:

- Frequency:

- Seven or more episodes in 1 year

- Five or more episodes per year for 2 years

- Three or more episodes per year for 3 years

- Associated with one or more of the following:

- Temperature $>38.3^{\circ} \mathrm{C}\left(101^{\circ} \mathrm{F}\right)$

- Cervical lymphadenopathy

- Tonsillar exudate

- Positive test for GABHS

- Chronic tonsillitis unresponsive to antimicrobial therapy

- Severe halitosis

- PTA (greater than one episode)

- PFAPA syndrome (periodic fever, aphthous ulcers, pharyngitis, cervical adenitis)

- PANS/PANDAS syndrome: a controversial indication (pediatric acute-onset neuropsychiatric syndrome/pediatric autoimmune neuropsychiatric disorders associated with streptococcal infection)

\section{Indication for Adenoidectomy Alone}

- Moderate to severe nasal obstruction with persistent symptoms

- Refractory chronic sinusitis

- Recurrent acute otitis media or otitis media with effusion in a child who had prior tympanostomy tubes that have now extruded (e.g., repeat surgery when indicated would 
consist of adenoidectomy plus myringotomy \pm insertion of ventilation tube) and is over 4 years of age

\section{Postsurgical Complications of Adenotonsillectomy}

- Anesthesia related

- Pain: moderate to severe lasting 7-14 days, requiring analgesia

- Hemorrhage:

- Bimodal timing

- $<24$ h postoperative: $\sim 2 \%$

- 5 to 7 days postoperative (sloughing of eschar): $3 \%$

- Dehydration secondary to decreased oral intake

- Halitosis (expected)

- Immediate postoperative airway obstruction (due to anesthesia, analgesia, or sleep apnea)

- Persistence of obstructive sleep apnea

- Velopharyngeal insufficiency (VPI):

- New onset or worsening of existing VPI.

- High-risk patients: cleft palate, submucous cleft palate, impaired baseline palatal movement (e.g., neurogenic), very large adenoid pad, velocardiofacial syndrome. Risk is decreased by conservative approach to surgery.

\section{Obstructive Sleep Apnea (OSA) Syndrome}

\section{Definition}

- Sleep-disordered breathing (SDB): a pathophysiologic continuum that includes snoring, upper airway resistance syndrome, obstructive hypopnea syndrome, and OSA.

- OSA: episodes of complete or partial upper airway obstruction during sleep, often resulting in gas exchange abnormalities and arousals. True OSA results in detrimental clinical sequelae such as failure to thrive, behavior problems, learning problems, and cardiovascular disease.

- Criteria for OSA: clinically relevant symptoms and an apnea hypopnea index (AHI) > 1 or hypoventilation $(\mathrm{CO} 2>50 \mathrm{mmHg}$ for $>25 \%$ total sleep time) as determined on polysomnogram (PSG)

\section{Etiology}

- OSA syndrome affects $2-10 \%$ of children; adenotonsillar hypertrophy as a primary driver of OSA is most common between ages 2 and 6 years of age.

\section{Clinical Presentation}

- Nocturnal—snoring, mouth breathing, pauses in breathing, enuresis, gasping or choking, restless/awakenings:

- Children may still have OSA without snoring.

- Daytime: inappropriate sleepiness, inattention/learning problems, hyperactivity/aggression, irritability.

- Children with OSA during infancy are more likely to have a risk factor other than adenotonsillar hypertrophy or obesity, such as craniofacial abnormality, neurologic problems, and genetic syndrome.

\section{Diagnosis}

- The American Academy of Pediatrics recommends screening by history (snoring, daytime symptoms) during well-child checks.

- Focused sleep history.

- Physical exam-vitals including blood pressure; craniofacial, oral, nasal, and oropharyngeal exams; growth curve; and BMI should be recorded:

- Adenotonsillar hypertrophy and obesity are the major causes in otherwise healthy children.

- Usually there is evidence of large tonsils, but small tonsils may cause symptoms during sleep; adenoids are best visualized by nasal endoscopy.

- Strongest risk factor for OSA in adolescence is obesity.

- If there's normal weight and small tonsils/ adenoids, otolaryngology will look for more rare causes of obstruction such as 
laryngeal masses, lingual tonsillar hypertrophy, macroglossia, abnormal pharyngeal tone, and nasoseptal obstruction.

- PSG_-gold standard for definitive diagnosis (also requires presence of clinical symptoms) and can help to inform treatment decisions and counsel caregivers:

- If there's clinical suspicion without highrisk features (see below), offer PSG rather than referral (otolaryngology, pulmonology, sleep medicine physician)

- If there are high-risk features, PSG in a facility experienced with children and referral are recommended:

- Obesity, craniofacial abnormalities, mucopolysaccharidoses, Prader-Willi syndrome, sickle cell disease, Down syndrome, neuromuscular disorders, congenital hypoventilation syndrome, etc.

- Classification based on AHI: mild OSA (1-4.9), moderate OSA (5-9.9), severe (greater than 10)

\section{Treatment}

- Tonsillectomy and adenoidectomy-otherwise healthy children with OSA and adenotonsillar hypertrophy:

- Consideration of clinical symptoms, OSA severity, comorbidities.

- Obese children may experience weight gain after surgery.

- Supportive care with watchful waiting - mild OSA, mild symptoms, and reevaluation in 6 months:

- Data based on Childhood Adenotonsillectomy Trial (CHAT) [13]

- Positive airway pressure therapy: minimal anatomic reason for obstruction, poor surgical candidate, need to stabilize/optimize the patient prior to surgery, persistent OSA after tonsillectomy/adenoidectomy

- Rapid maxillary expansion

\section{MOUTH AND ORAL CAVITY}

\section{Aphthous Ulcers}

- Aphthous ulcer is the most common oral ulcer.

\section{Etiology}

- Idiopathic (most common); other causes include immune disorders, infections, hormonal cause, stress, trauma, and nutrition.

- Painful white ulcers on keratinized gingiva surrounded by erythematous border.

Types

- Minor: most common, $<1 \mathrm{~cm}$ in diameter, painful, burning/tingling prodrome

- Major: more painful, 1-3 cm in diameter, 1 to 10 ulcers at one time, scarring potential

- Herpetiform: multiple small ulcers (1-3 mm in diameter)

- Sutton's disease: recurrent aphthous ulcers (major type)

\section{Treatment}

- Observation (self-limiting course)

- May also consider analgesia, anti-inflammatories, antibiotics if superinfected, and antivirals

\section{Bifid Uvula}

- $2 \%$ of population (up to $10 \%$ in some ethnic groups)

- Clinical presentation ranges from split to duplication.

- May be a marker of submucous cleft palate:

- Lucency of the midline soft palate, palpable notch of the midline hard palate

- Associated with speech problems, VPI, dysphagia

- Rarely, may be associated with genetic syndromes: 
- Cornelia de Lange syndrome

- Loeys-Dietz syndrome: increased risk of aortic aneurysm

\section{Cold Panniculitis}

- Lobular, erythematous, acute eruption in adipose tissue following exposure to cold and subsequent inflammation.

- Cheeks and forehead often affected in infants.

- Differential: adiponecrosis subcutanea (subcutaneous fat necrosis of the newborn, cold panniculitis of the newborn), sclerema neonatorum (often fatal, needle-shaped crystals), post-steroid panniculitis, frostbite, and group A streptococcal bacteremia.

- Diagnosis is clinical; biopsy if needed will show lobular panniculitis with occasional deposition of mucin.

- Treatment is symptomatic, with slow rewarming.

\section{Ankyloglossia}

- Abnormally short frenulum limiting effective tongue mobility

- In infants, if severe, may present with suckling difficulties and painful latch (if breastfeeding)

- In older children, may result in speech articulation issues, social mechanical issues (i.e., difficulty in licking an ice-cream cone, keeping teeth clean, playing wind instruments, "French" kissing)

- Surgical intervention indicated for problematic symptoms

\section{Mucocele}

- Painless, bluish submucosal lesion appearing on the lower lip (Fig. 18.11)

- Typically secondary to trauma (i.e., biting lower lip)

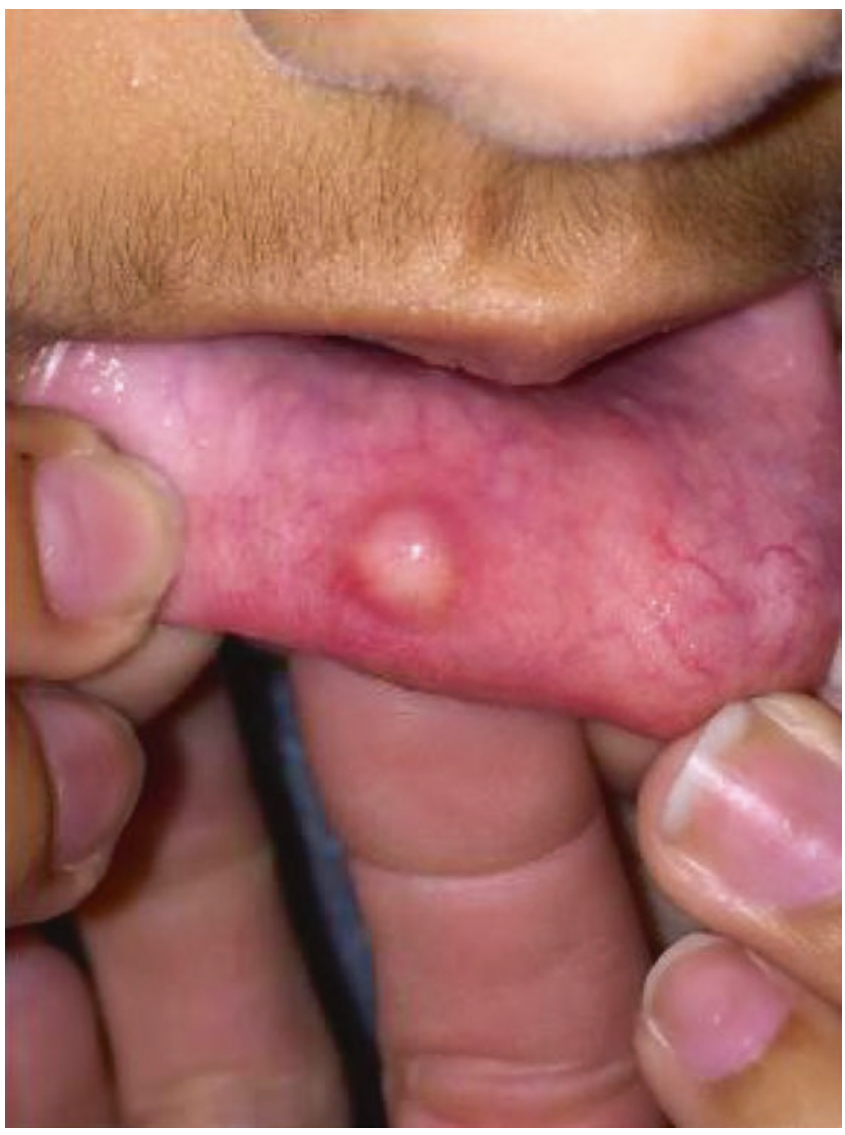

Fig. 18.11 Mucocele of the lower lip

- Can slowly grow in size

- Treatment: observation if not bothersome, surgical excision

\section{Parotitis}

\section{Etiology}

- Salivary stasis, obstruction (usually unilateral; stone, mass/tumor), retrograde bacterial migration, idiopathic

- Bacteria: Staphylococcus aureus (most common), Streptococcus viridans, H. influenzae, Streptococcus pyogenes, E. coli

- Viruses (usually bilateral): HIV, mumps, influenza, coxsackie

- Recurrent parotitis of childhood:

- Unknown etiology

- Episodes occurring every 1-3 months

- May alternate sides 
- Typically resolves spontaneously

- No antibiotic therapy needed unless there's presence of systemic symptoms

\section{Symptoms}

- Tender, red, warm parotid gland

- Purulence at Stensen's duct with "milking” of gland

\section{Diagnosis}

- History and physical exam

- Cultures of purulent discharge to help guide antibiotic therapy

\section{Treatment}

- Conservative: rehydration, warm compresses, parotid massage, sialogogues.

- Antibiotics (based on cultures)

- If there's no improvement with above treatment, consider parotid imaging (CT or ultrasound) for evaluation of abscess, stone, or obstructing lesion.

\section{Cleft Lip and Palate (CLP)}

\section{Epidemiology}

- Second most common malformation (after clubfoot)

- Cleft lip and palate (CLP): 1/1000 births.

- Cleft palate (CP): 1/2000 births (Fig. 18.12)

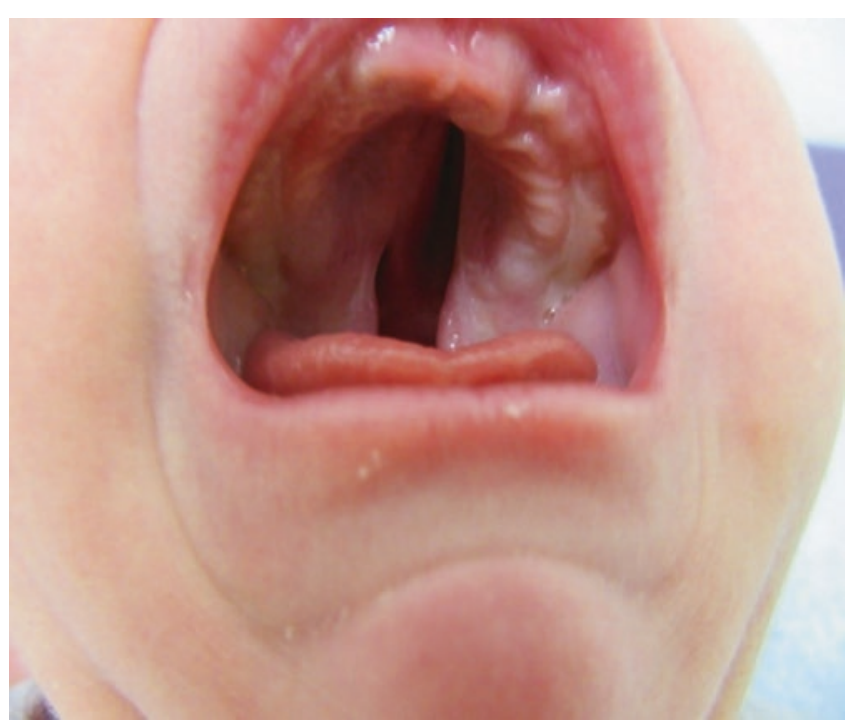

Fig. 18.12 Young patient with cleft palate
- Cleft lips (+/- cleft palate) and isolated cleft palate occur in distinct genetic lines.

- Higher prevalence in Asians and Native Americans.

- Cleft lip (CL): Males > females.

- Isolated cleft palate: Females > males.

Risk Factors

- Teratogens (ethanol, thalidomide)

- Maternal diabetes

- Amniotic band syndrome

\section{Genetic Evaluation}

- $8 \%$ of isolated cleft palates are associated with a syndrome.

- Over 200 syndromes are associated with CL/ CLP, which most commonly include:

- Stickler syndrome: CP, retinal detachment, cataracts

- Treacher Collins syndrome: CP, midface hypoplasia, eyelid colobomas, ossicular abnormalities

- Apert syndrome: CP, acrocephaly, fused digits, stapes fixation

\section{Feeding Difficulties}

- Infants experience difficulty with "seal"often requires specialized nipple (i.e., Mead Johnson crosscut, McGovern's nipples)

- Often require feeding in more upright position with frequent rests and burping.

\section{Otologic Disease}

- Increased risk of developing Eustachian tube dysfunction resulting in OME with CP/CLP

- Often requires myringotomy/ventilation tubes

\section{Timing of Surgical Intervention}

- CL generally is surgically repaired between the ages of 10 and 12 weeks:

- "Rule of tens"-10 pounds, 10 weeks old, and hemoglobin of $10.0 \mathrm{~g} / \mathrm{dL}(100.0 \mathrm{~g} / \mathrm{L})$

- $\mathrm{CP}$ is usually repaired between 9 and 12 months of age.

\section{For Follow-Up}

- Difficulty in feeding and growth

- Recurrent ear infections/possible hearing loss 
- Dysfunctional speech and communication (i.e., velopharyngeal dysfunction)

- Dental problems

- Social struggles because of the child's appearance

\section{Robin Sequence (RS)}

- Sequence defined as micrognathia, cleft palate, and glossoptosis.

- Occurs in isolation or with associated syndrome (i.e., trisomy 18 or Stickler syndrome)

- Infants with RS are at high risk to develop respiratory distress and potentially have "difficult airways," given their anatomy. These infants require close airway monitoring in the postnatal period.

- Management of respiratory distress in RS:

- Prone positioning.

- Place suture at the tip of the tongue and pull the tongue forward.

- Intubate if needed. If unable to intubate, place a laryngeal mask airway.

- If patient fails extubation, patient may require:

- Mandibular distraction

- Tracheostomy

\section{Delayed Dental Eruption}

- Normal range for dental eruption is between 8 and 18 months.

- Delayed dental eruption is considered when teeth fail to erupt within 12 months of "normal range."

- Possible etiologies include hypothyroidism, hypopituitarism, ectodermal dysplasia, and rickets.

\section{Odontogenic Infection}

Etiology

- Caries are typically the primary cause of odontogenic infections.
- Polymicrobial:

- Streptococcus mutans (most common cause of initial caries infection)

- Alpha-hemolytic streptococci

- Anaerobes (Peptostreptococcus, Bacteroides, Fusobacterium)

\section{Clinical Presentation}

- Localized pain, edema, erythema, purulence

- Sensitivity to temperatures and palpation, loose tooth

- Orofacial swelling:

- Swelling below the jaw (mandibular abscess)

- Periorbital swelling (maxillary abscess)

\section{Imaging}

- Evaluate airway compromise, gas-producing organisms, presence of abscess, and extent of involvement.

- Panorex.

- CT scan.

\section{Treatment}

- Remove source of infection (i.e., tooth)

- Analgesia.

- Antibiotics.

- Incision and drainage if an abscess is present.

\section{Early Childhood Caries}

\section{Definition}

- Caries affecting the primary dentition, especially in the first 3 years of life

\section{Caries Formation}

- Chronic infectious disease.

- Pathogenesis: Tooth-adherent bacteria (most commonly Streptococcus mutans) metabolize sugars to produce acid that leads to demineralization of the tooth structure.

\section{Risk Factors}

- Bottle propping (affects predominantly central incisors) 
- Low-income households

- Excessive consumption of sugar

- Genetic factors

\section{Prevention}

- Dental visit within the first 6 months of first tooth eruption and no later than 1 year of age.

- Tooth brushing is suggested twice daily with an age-appropriate size of fluoridated toothpaste (discourage swallowing toothpaste to prevent fluorosis)

- Avoid high-frequency consumption of highsugar liquids/solid foods.

- Recommend weaning from bottle between 12 and 18 months and transitioning to a cup.

\section{Fluoride Supplementation}

- Dental fluorosis occurs during the development of the tooth (critical ages between 0 and 6 years of age, with most important being between 15 and 30 months) [14]

- Be aware that access to fluoridated water may be limited in some areas in the USA.

- Optimal water fluoridation is $0.7 \mathrm{ppm}$ of fluoride.

- If there's limited access to fluoridated water, supplementation may be considered, especially for patients between 15 and 30 months of age.

\section{Dental Trauma and Avulsions}

\section{Primary Tooth Avulsion}

- Refer to the dentist for follow-up to rule out any associated problems.

- Avoid reimplantation of primary avulsed tooth.

- Permanent tooth avulsion (it is a true dental emergency) [15]:

- Reimplantation of tooth:

o If reimplanted within $5 \mathrm{~min}$, tooth survival rate is $85-97 \%$

- If reimplanted after $1 \mathrm{~h}$ of injury, tooth is unlikely to survive.

- Instructions for avulsed permanent tooth:

- Gently wash the avulsed tooth with no rubbing or brushing.
- Reimplant the tooth into the socket as soon as possible.

- If not possible, preserve the tooth in saliva, milk, or normal saline.

- The goal is to maintain viability of the periodontal ligament fibers.

- Child should be transported to a dental office or the nearest emergency room.

\section{NECK}

\section{Cervical Lymphadenitis}

Pathogens

- Viral: EBV (most common virus), CMV, HSV, adenovirus, enterovirus, roseola, rubella, HIV

- Bacterial: group A Streptococcus (most common), Staphylococcus aureus

\section{Clinical Presentation}

- Fevers (typically low grade for viral), malaise, tender and mobile cervical nodes (Fig. 18.13)

\section{Diagnosis}

- History and physical examination

- Possible aspiration for culture and sensitivity

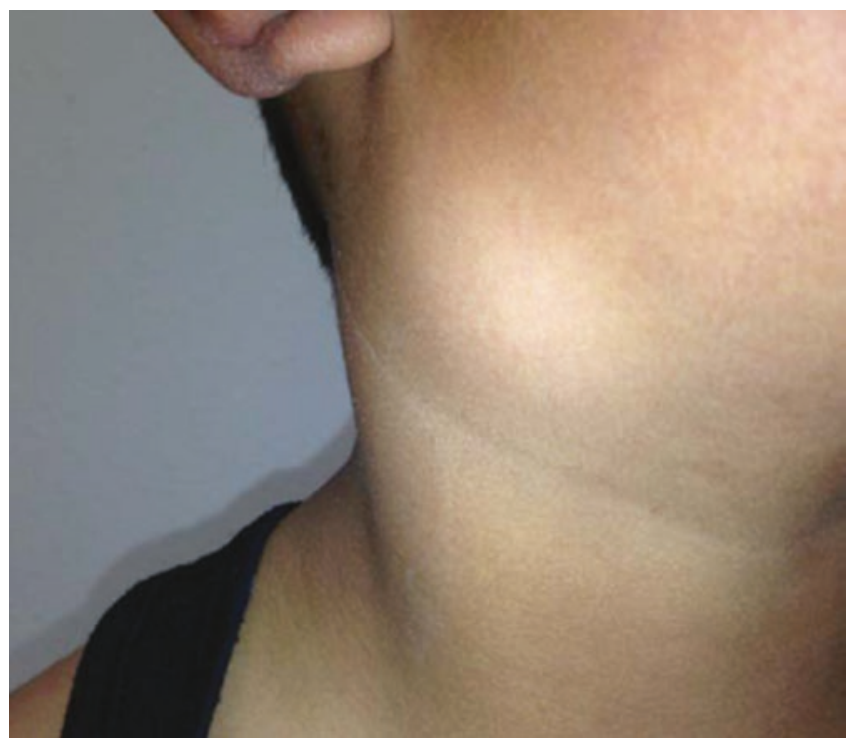

Fig. 18.13 9-year-old boy presenting with a high fever of $104^{\circ} \mathrm{F}$, malaise, and tender large bacterial cervical lymphadenopathy 


\section{Complications}

- Cellulitis, abscess, internal jugular vein thrombosis, mediastinitis, sepsis

\section{Treatment}

- Viral: supportive

- Bacterial:

- Antibiotics

- Incision and drainage if there is an abscess formation

\section{Infectious Mononucleosis}

- Caused by EBV

\section{Clinical Presentation}

- Fever, pharyngitis, and lymphadenopathy.

- Symmetric cervical adenopathy (most commonly, posterior triangle nodes)

- Axillary and inguinal nodes may also be involved.

- Fatigue, malaise, splenomegaly.

\section{Diagnostic Tests}

- Monospot test ("heterophile antibody"):

- High false-negative rate if obtained early on in illness or in children under 4 years of age

- Elevated immunoglobulin M titer to viral capsid antigen (IgM-VCA) indicates acute infection.

\section{Cat Scratch Disease}

- Pathogen: Bartonella henselae

\section{Clinical Presentation}

- Present 2 weeks after cat scratch or bite (usually from a kitten)

- Papular lesion at primary scratch site associated with cervical lymphadenopathy (tender initially and then becomes painless)—may ulcerate and form fistula
- Fever (often mild), malaise

Diagnosis

- Serology (IgG henselae titers), culture (Warthin-Starry stain), polymerase chain reaction (PCR) assay, histopathology

\section{Treatment}

- Supportive (typically self-limiting)

- Antibiotic therapy in immunocompromised patients

- Surgical aspiration for culture, but avoid formal incision and drainage to prevent fistula/ sinus formation

\section{Atypical Mycobacteria}

- Pathogen: Mycobacterium avium complex, M. scrofulaceum, M. kansasii

\section{Risk Factors}

- Young children, immunocompromised.

- If patient is less than 1 year old, consider alternative diagnosis such as Langerhans histiocytosis.

\section{Clinical Presentation}

- Asymptomatic.

- Unilateral cervical lymphadenopathy, preauricular adenopathy, commonly located on the face over body of mandible.

- Adhesive to overlying skin; overlying skin is erythematous in advanced disease.

- Lesions often described as having "violaceous" coloring.

\section{Diagnosis}

- Acid-fast stain, culture (requires 2-4 weeks for results)

\section{Treatment}

- Watchful waiting (typically takes months to resolve)

- Excision or incision and curettage (avoid incision and drainage, as fistula can result) 


\section{Other Causes, Lymphadenitis}

- Tuberculosis: in children, less common than atypical mycobacterium

- Kawasaki disease (mucocutaneous lymph node syndrome):

- Acute vasculitis affecting multiple organs in children

- Diagnosis:

- Must have five of the following:

- Fever $>5$ days (high)_absolute criteria

- Erythematous rash

- Conjunctival injection

- Oropharyngeal changes

- Peripheral extremity changes (induration or desquamation)

- Cervical lymphadenopathy

- Echocardiogram

- High risk of developing coronary aneurysm or myocardial infarction (consider IVIG and aspirin therapy, along with EKG and echo)

\section{Kikuchi}

- Rare disease of unknown etiology

- Presentation: young women, cervical and generalized lymphadenopathy, fever, night sweats, rash, weight loss, nausea, and vomiting

- Diagnosis: lymph node biopsy-histiocytic necrotizing lymphadenitis

- Treatment:

- No effective treatment, typically resolves within 1-4 months.

- Symptom control with steroids.

- Follow-up is necessary, as patients with Kikuchi are at higher risk of developing systemic lupus.

\section{Tularemia}

- Pathogen: Francisella tularensis

- Transmission: contact with infected animal (i.e., rabbits or hamsters)
- Presentation: febrile illness, ulceroglandular syndrome (painful regional lymphadenopathy and an ulcerated skin lesion)

- Treatment: streptomycin

\section{Castleman's Disease}

- Lymphoproliferative disorder localized to a single node (unicentric) or systemically (multicentric)

- Polyclonal proliferation of B-lymphocytes

- Unicentric:

- Typically asymptomatic - presents with an enlarged lymph node ( $20 \%$ in the neck)

- CT scan shows a well-circumscribed mass.

- Pathology demonstrates nodal expansion.

- Surgical removal is curative $90 \%$ of the time.

- Multicentric:

- May be associated with HIV, Kaposi sarcoma-associated herpesvirus, and/or human herpesvirus type 8 .

- No standard treatment. May include antivirals, chemotherapy, corticosteroids, and monoclonal antibodies.

- Refer to oncology.

\section{Lymphoma}

- Most common pediatric malignancy of the head and neck.

- Lymphoproliferative disorder.

- Hodgkin and non-Hodgkin lymphoma may present with cervical lymphadenopathy.

\section{Clinical Presentation}

- Nodal masses-may present with cervical nodes

- Hodgkin: contiguous lymph nodes

- Non-Hodgkin lymphoma: may present with extranodal involvement (i.e., enlarged tonsils, base of tongue, enlarged thyroid, etc.)

- Constitutional symptoms: fevers, night sweats, weight loss 


\section{Diagnosis}

- History and physical examination

- Evaluation of all nodal sites

- Open biopsy (rather than fine needle biopsy)_fresh tissue is required for immunochemistry

\section{Management}

- If positive for lymphoma, refer to oncology.

\section{Thyroglossal Cyst}

\section{Definition}

- Failed obliteration of the thyroglossal duct (embryologic tract from the foramen cecum of the tongue to the thyroid)

\section{Clinical Presentation}

- Midline neck mass (often cystic, may be solid, mixed, or inflamed)_-surrounding the hyoid bone and superior to the thyroid (Fig. 18.14)

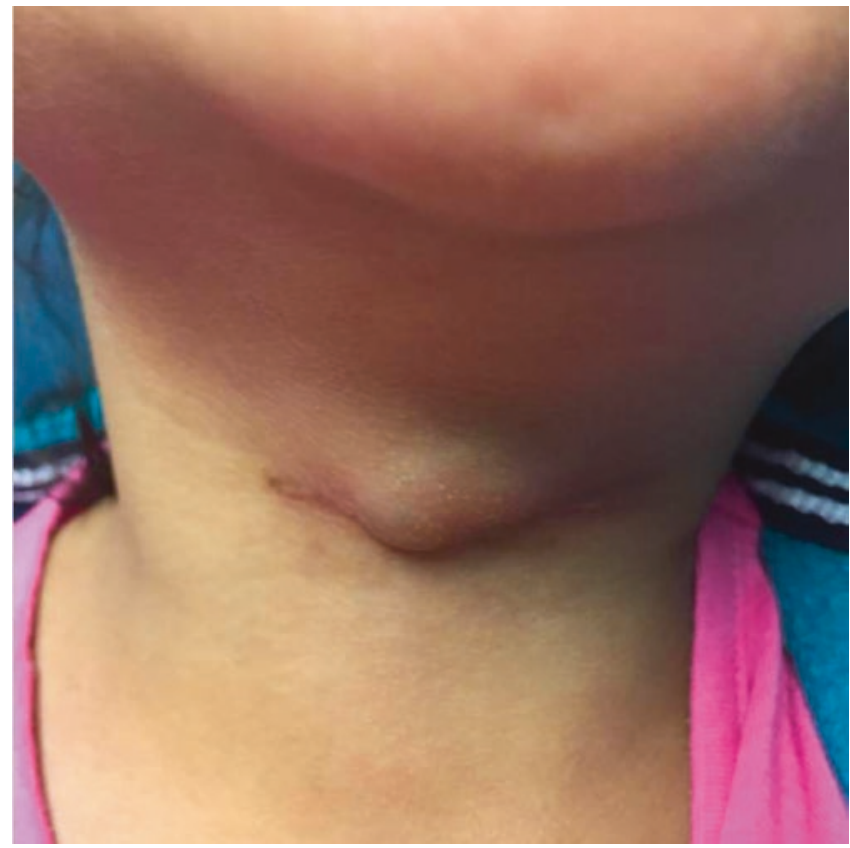

Fig. 18.14 Typical appearance of a neck mass associated with underlying thyroglossal duct cyst. It is important to obtain an ultrasound of the neck to confirm the presence of a normal thyroid gland prior to removal of the thyroglossal duct cyst in order to avoid inadvertent removal of the only thyroid tissue
- Elevates with tongue protrusion (pathognomonic)

\section{Complications}

- May become infected

- Rare malignant potential

\section{Treatment}

- Treat infection with antibiotics (avoid incision and drainage)

- Surgical removal when not infected (Sistrunk procedure)

\section{Branchial Cleft Cyst}

- Alterations of the branchial apparatus resulting in cysts, sinuses, or fistula

\section{Presentation}

- Unilateral (most common)

- Anterior, lateral neck mass (typically anterior to sternocleidomastoid muscle), sinus, or fistula

- May become infected with drainage (associated with URI)

\section{Treatment}

- Treat infection with antibiotics (avoid incision and drainage)

- Complete surgical excision of cyst, sinus, and fistula tract once infection resolves.

\section{Lymphatic Malformation}

- Also known as cystic hygroma and lymphangioma (outdated terms)

- Etiology: abnormal lymphatic development

\section{Presentation}

- May occur anywhere in the body

- Soft, painless, multiloculated, compressible mass that transilluminates:

- In the cervical region, posterior triangle is most common.

- Present at birth or shortly thereafter:

- Often enlarges after URI 
- Associated symptoms related to mass compression of nearby structures

Imaging: MRI preferred

\section{Management}

- Observation if small and with no associated complications

- Sclerosing agents

- Surgical excision

\section{Thyroid Carcinoma}

\section{Presentation}

- Midline anterior mass, may have associated lateral neck masses (lymph nodes):

- Painless, solid, fixed, asymptomatic mass.

- Thyroid nodules in children reportedly have a $\sim 25 \%$ incidence of malignancy.

- Neck metastasis is frequently the presenting symptom.

- More common in females (2 to 3 times), adolescents, and those with personal history of autoimmune thyroid disease.

- History should include radiation exposure and family history.

- May be associated with genetic syndromes:

- Multiple endocrine neoplasia type 2 (MEN2) syndromes: medullary thyroid carcinoma (MTC):

- MEN2A: MTC, pheochromocytoma, parathyroid adenoma, or hyperplasia

- MEN2B: MTC, pheochromocytoma, Marfanoid habitus, mucosal neuromas

- Familial medullary thyroid cancer: MTC only, affects 5-35\% of MEN2 families

- Familial adenomatous polyposis: papillary form, may be in younger age

- Cowden syndrome (PTEN hamartoma tumor syndrome): papillary or follicular form

- Associated symptoms related to mass compression (dysphagia, dyspnea) or invasion of nearby structures (hoarseness) are very rare in children.

\section{Workup}

- Ultrasound of the thyroid and neck preferred as initial imaging, often with fine needle aspiration (plus molecular studies) of thyroid mass

- Thyroid function tests and thyroid antibody studies (in consultation with endocrinology)

- Complete metabolic panel (including calcium)

- Chest radiograph (lung metastasis, most common site of distant disease)

\section{Pathology}

- Papillary (most common), follicular, medullary, anaplastic

\section{Management}

- Multidisciplinary care team referral (endocrinology, genetics, otolaryngology/pediatric surgery)

- Treatment is surgical, with additional therapy pending the pathology results and patient factors.

\section{Acute Laryngitis}

\section{Etiology}

- Infectious (most commonly viral, may have secondary bacterial infection)

- Fungal infection (immunocompromised child, steroid inhaler use)

- Vocal strain (secondary to screaming/ yelling)

\section{Management}

- Generally self-limiting.

- Optimize hydration.

- Humidification.

- Saltwater gargles.

- Treat with antibiotics or antifungals if bacterial or fungal infection is suspected.

- Referral to otolaryngology if it persists $>4$ weeks. 


\section{Chronic Laryngitis/Hoarseness}

- Definition: symptoms of hoarseness, dysphonia, and/or vocal fatigue for $>3$ months

- Associated symptoms: chronic cough, frequent throat clearing, postnasal drip sensation

\section{Etiology}

- Typically noninfectious causes (most common vocal fold screamers' nodules)

- Gastroesophageal reflux disease/laryngopharyngeal reflux disease

- Recurrent respiratory papillomatosis (caused by HPV 6 and HPV 11)

- Environmental irritants (second-hand smoke, vape, huffing)

- Environmental allergies

- Postnasal drip/rhinitis

- Medications (e.g., inhaled steroids, chemotherapy, anti-cholinergic medications)

- Rarely, chronic systemic disease (e.g., amyloid, granulomatosis with polyangiitis), lymphovascular malformation, or malignancy

\section{Diagnosis}

- ENT referral for flexible nasolaryngoscopy

\section{Management}

- Treat underlying cause.

- Empiric treatment of GERD/ laryngopharyngeal reflux (LR) is often considered if clinical history is suggestive.

\section{Vocal Fold Paralysis}

\section{Background}

- One of the most common laryngeal abnormalities in childhood

- Unilateral or bilateral paralysis of the vocal fold

- Congenital or acquired

\section{Etiology}

- Iatrogenic (most common in pediatric patients): patent ductus arteriosus ligation, cardiothoracic surgery, tracheoesophageal fistula repair, thyroidectomy

- Idiopathic (most common in newborns/ infants)

- Neurologic (e.g., Arnold-Chiari malformation, posterior fossa tumor)

- Viral

- Autoimmune

- Pulmonary lesion or cor pulmonale (Ortner's syndrome)

\section{Diagnosis}

- Refer to ENT for flexible laryngoscope, which will assess for vocal fold mobility, mucosal lesions, and laryngeal masses.

\section{Workup of Vocal Fold Paralysis}

- Observation (if known iatrogenic cause)

- Chest radiograph

- Modified barium swallow (to assess for aspiration)

- MRI brain

- CT for the neck and chest

\section{Treatment}

- Observation:

- Monitor for signs of aspiration or respiratory distress.

- Monitor for signs of recovery.

- Surgery:

- Tracheostomy (bilateral)

- Vocal fold surgery

- Hypoglossal to recurrent laryngeal nerve reanastomosis

\section{Stridor}

- Noisy breathing, which often implies turbulent airflow through an extrathoracic airway obstruction 
- Stertor: Distinct from stridor. Stertor is more often a muffled, congested upper airway noise emanating from the nose, nasopharynx, or oropharynx.

\section{Etiology}

- Inspiratory: most often above the level of the vocal folds

- Expiratory: most often in the trachea

- Biphasic: most often at the level of the vocal folds (glottis) or the subglottis

\section{Differential Diagnosis}

- Acute-most often of infectious or inflammatory cause: number 1 , croup (parainfluenza virus); influenza virus type A or B, RSV, rhinovirus, epiglottitis, bacterial tracheitis, angioedema, foreign body

- Chronic-most often of an anatomic cause: number 1, laryngomalacia; vocal fold immobility (unilateral or bilateral), vascular ring, tracheomalacia, papilloma, subglottic stenosis, laryngeal web, tracheal stenosis, subglottic hemangioma

\section{Diagnosis}

- History: Symptoms related to positioning, feeding; apparent life-threatening events (ALTEs)/brief, resolved, unexplained events (BRUEs), cyanotic episodes, crying/agitation, and trajectory are especially important.

- Physical exam: vitals including pulse oximetry, growth curve, complete head and neck evaluation; cardiopulmonary evaluation including inspection for retractions, abdominal breathing, respiratory distress.

- ENT referral for flexible nasolaryngoscopy and further evaluation.

- If pathology is thought to be below the level of the vocal folds (e.g., subglottic stenosis), operative laryngoscopy and bronchoscopy are also needed to evaluate the airway.

- If there's suspicion of vascular ring, refer to cardiology and consider of CT angiography of the chest.

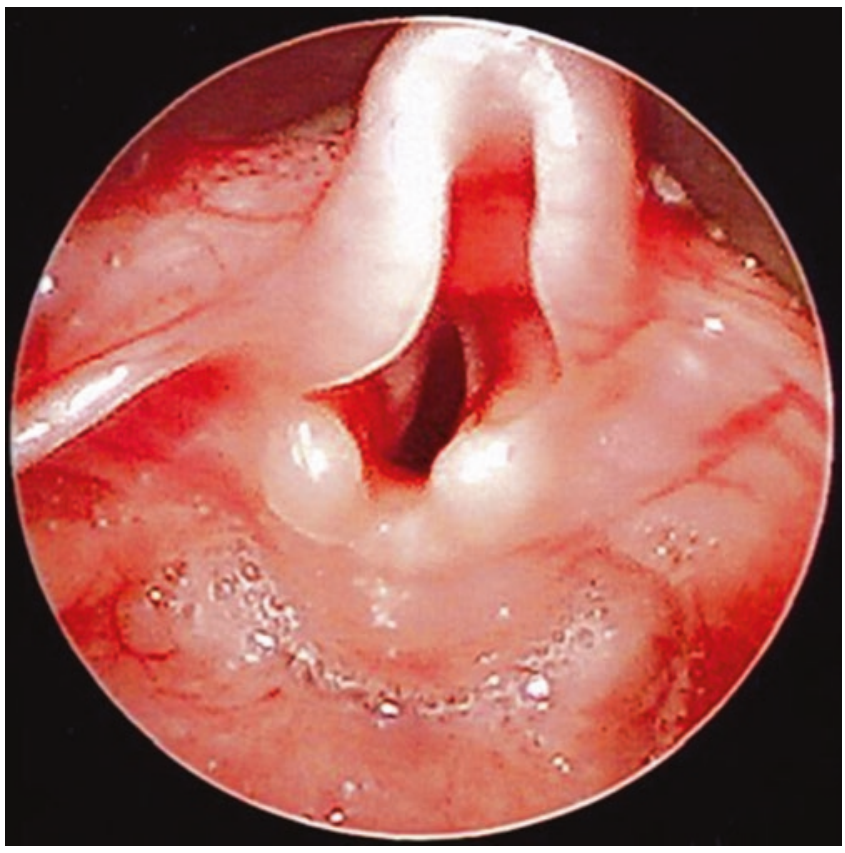

Fig. 18.15 Mild to moderate laryngomalacia as evidenced by tubular ("omega-shaped") epiglottis, knobby, or bulky arytenoid cartilages and tight aryepiglottic folds

\section{Management}

- Treat underlying cause.

- Empiric treatment of GERD/LPR often considered if clinical history is suggestive.

\section{Laryngomalacia}

- Collapse of the supraglottic airway (epiglottis, arytenoid cartilages, or both) during inspiration secondary to immature framework (Fig. 18.15)

- Most common congenital lesion of the larynx.

- High-pitched inspiratory stridor usually begins around 4-6 weeks, peaks around 6-8 months, and resolves by 24 months of age.

- Higher incidence of GERD

\section{Symptoms}

- Stridor, feeding difficulty, sleeping difficulty, need for frequent repositioning (increased when supine), increased work of breathing/ retractions, failure to thrive, cyanotic episodes (rare); may worsen in the setting of URI 


\section{Management}

- $90 \%$ of patients treated with conservative management and time; referral to ENT for flexible nasolaryngoscopy and consideration of surgical management (airway evaluation, possible supraglottoplasty) if symptoms are moderate/severe

- Surgery considered for failure to thrive, hypoxemia, severe retractions, sleep apnea

\section{PEARLS AND PITFALLS}

- Otalgia is an expected phenomenon for up to 2 weeks following tonsillectomy.

- Hematoma of the external ear (pinna) necessitates same-day referral for emergency care because of the potential for permanent deformity secondary to avascular necrosis of the cartilage.

- First-line therapy for AOM is amoxicillin $(90 \mathrm{mg} / \mathrm{kg} /$ day divided twice a day $) \times 10$ days .

- Caregivers should be instructed to warm ear drops in their hands prior to administration to decrease patient discomfort.

- Patients with benign paroxysmal vertigo of childhood are at increased risk of typical migraine headache as adolescents and adults.

- CHARGE is the most commonly associated congenital anomaly with choanal atresia.

- In children, $90 \%$ of epistaxis occurs from the anterior septum (Kiesselbach plexus), and the most frequent cause is digital trauma.

- Nasal fractures are the most common facial fracture in children.

- Presence of nasal polyps in children should prompt testing for cystic fibrosis.

- Nasal saline rinses should be used with caution in children with history of aspiration.

- The most common cause of a neck mass in the pediatric population is cervical lymphadenitis.

- If there is clinical suspicion for lymphoma, systemic steroids should be avoided, as these may interfere with flow cytometry results.
- Midline neck mass is most likely a thyroglossal duct cyst secondary to the embryologic derivative at the base of the tongue (foramen cecum). Ultrasound should be performed to confirm the presence of a normal thyroid in its expected location.

- The most common congenital lesion of the larynx is laryngomalacia; most children will outgrow the diagnosis by 24 months of age.

- Cough, rhinorrhea, and diarrhea are more common with viral than with bacterial pharyngitis.

- The diagnostic gold standard for bacterial pharyngitis is a throat culture.

- Diagnosis of PTA is a clinical diagnosis based on history (double worsening, URI symptoms $>5$ days prior to new symptoms) and physical exam (hot potato voice, trismus, uvular deviation)

- The American Academy of Pediatrics recommends screening for OSA by history (snoring, daytime symptoms) during well-child checks. Symptoms may include irritability, hyperactivity, daytime sleepiness, and nocturnal enuresis; this is a different constellation of symptoms than in adult patients.

- Ankyloglossia often manifests as discomfort in the mother's nipples.

- Children with cleft palate are at an increased risk of developing Eustachian tube dysfunction resulting in OME and recurrent AOM.

- Eruption cysts present as blue or purple compressible cysts at the site of an erupting deciduous or permanent tooth. These are often self-limiting but may require treatment if they become infected or limit feeding.

Acknowledgement The authors gratefully acknowledge the contributions of Josee Paradis, MD, Paediatric Surgery, London Health Sciences Centre-Victoria Hospital, London, Ontario, Canada, to the first edition of this chapter, many of which have been incorporated into this edition as well. 


\section{References}

1. Wang RY, Earl DL, Ruder R, Graham JM Jr. Syndromic ear anomalies and renal ultrasounds. Pediatrics. 2001;108(2):E32.

2. Uhari M, Mantysaari K, Niemela M. A meta-analytic review of the risk factors for acute otitis media. Clin Infect Dis. 1996;22(6):1079.

3. Lieberthal AS, Carroll AE, Chonmaitree T, Ganiats TG, Hoberman A, Jackson MA, et al. The diagnosis and management of acute otitis media. Pediatrics. 2013;131(3):e964-99. Erratum Pediatrics. 2014;133(2):346. Dosage error in article text.

4. Rosenfeld RM, Shin JJ, Schwartz SR, Coggins R, Gagnon L, Hackell JM, et al. Clinical practice guideline: otitis media with effusion (update). Otolaryngol Head Neck Surg. 2016;154(1 Suppl):S1-S41.

5. Rosenfeld RM, Schwartz SR, Pynnonen MA, Tunkel DE, Hussey HM, Fichera JS, et al. Clinical practice guideline: tympanostomy tubes in children. Otolaryngol Head Neck Surg. 2013;149(1 Suppl):S1-S35.

6. Alford RL, Arnos KS, Fox M, Lin JW, Palmer CG, Pandya A, ACMG Working Group on Update of Genetics Evaluation Guidelines for the Etiologic Diagnosis of Congenital Hearing Loss; Professional Practice and Guidelines Committee, et al. American College of Medical Genetics and Genomics guideline for the clinical evaluation and etiologic diagnosis of hearing loss. Genet Med. 2014;16(4):347-55.

7. Wald ER, Applegate KE, Bordley C, Darrow DH, Glode MP, Marcy SM, American Academy of Pediatrics, et al. Clinical practice guideline for the diagnosis and management of acute bacterial sinusitis in children aged 1 to 18 years. Pediatrics. 2013;132(1):e262-80.
8. Chow AW, Benninger MS, Brook I, Brozek JL, Goldstein EJ, Hicks LA, et al. Infectious Diseases Society of America. IDSA clinical practice guideline for acute bacterial rhinosinusitis in children and adults. Clin Infect Dis. 2012;54(8):e72-e112.

9. Choby BA. Diagnosis and treatment of streptococcal pharyngitis. Am Fam Physician. 2009;79(5):383-90.

10. Stewart EH, Davis B, Clemans-Taylor BL, Littenberg B, Estrada CA, Centor RM. Rapid antigen group a streptococcus test to diagnose pharyngitis: a systematic review and metaanalysis. PLoS One. 2014;9(11):e111727.

11. Baugh RF, Archer SM, Mitchell RB, Rosenfeld RM, Amin R, Burns JJ, American Academy of Otolaryngology-Head and Neck Surgery Foundation, et al. Clinical practice guideline: tonsillectomy in children. Otolaryngol Head Neck Surg. 2011;144(1 Suppl):S1-30.

12. Ramos SD, Mukerji S, Pine HS. Tonsillectomy and adenoidectomy. Pediatr Clin N Am. 2013;60(4):793-807.

13. Marcus CL, Moore RH, Rosen CL, Giordani B, Garetz SL, Taylor HG, Childhood Adenotonsillectomy Trial (CHAT), et al. A randomized trial of adenotonsillectomy for childhood sleep apnea. N Engl J Med. 2013;368(25):2366-76.

14. Hong L, Levy SM, Broffitt B, Warren JJ, Kanellis MJ, Wefel JS, Dawson DV. Timing of fluoride intake in relation to development of fluorosis on maxillary central incisors. Community Dent Oral Epidemiol. 2006;34(4):299-309.

15. Andersson L, Andreasen JO, Day P, Heithersay G, Trope M, Diangelis AJ, et al. International Association of Dental Traumatology guidelines for the management of traumatic dental injuries: avulsion of permanent teeth. Dental Traumatol. 2012;28(2):88-96. 